\title{
Magnetic resonance imaging of the hip: anatomy and pathology
}

\author{
May San Mak ${ }^{\mathrm{EF}}$, James Teh ${ }^{\mathrm{E}, \mathrm{F}}$ \\ Department of Radiology, Nuffield Orthopaedic Centre, Oxford University Hospitals NHS Trust, UK
}

\section{Abstract}

The aim of this review is to outline the normal anatomy of the hip and to discuss common painful conditions of the hip that affect the general adult population. Hip pain is a common complaint with many different etiologies. In this review, hip pathologies are divided by location into osseous, intra-articular and extra-articular lesions. Magnetic resonance imaging (MRI) is the modality of choice for investigating painful hip conditions due to its multiplanar capability and high contrast resolution. This review focuses on the characteristic MRI features of common traumatic and pathologic conditions of the hip.

Key words: femoroacetabular impingement, MRI hip, acetabular labral tear, hip arthritis, greater trochanteric pain syndrome, extra-articular pathology.

\section{Introduction}

Hip pain is a common affliction with many different causes. The hip joint can be difficult to examine clinically due its deep location; therefore, imaging represents a key diagnostic tool for hip conditions [1]. Magnetic resonance imaging (MRI) with its multiplanar capability, high contrast resolution, and lack of ionising radiation is an excellent modality for evaluating painful conditions of the hip [2]. Detailed knowledge of hip anatomy is essential in understanding its pathologies. In this article, the relevant components of hip anatomy, including the osseous structures, articular hyaline cartilage, fibrocartilaginous acetabular labrum, joint capsule, and ligaments, as well as surrounding tendons and bursae, are reviewed. Frequently encountered anatomical variants and asymptomatic findings are also described. In this article, the authors review the role of MRI in diagnosing common conditions causing hip pain and their characteristic features.

\section{Anatomy of the hip}

\section{Bones}

The hip is a weight-bearing synovial joint that is inherently stable yet allows for a wide range of motion. It is a highly congruent ball-and-socket joint consisting of the femoral head (ball) articulating with the acetabulum (socket). The bony acetabulum is an incomplete spherical cavity with an inferior depression which is the acetabular notch. The transverse acetabular ligament bridges the acetabular notch and completes the acetabular socket [1]. There is a spectrum of femoral head and acetabular morphologies. Deviation from the normal morphology with resultant poor osseous congruency is associated with conditions such as congenital hip dysplasia and femoroacetabular impingement (FAI) [3]. On MRI, the normal femoral head of adults demonstrates homogeneous high signal on T1-weighted sequences because of fatty marrow

\footnotetext{
Correspondence address:

James Teh, Nuffield Orthopaedic Centre, Oxford University Hospitals NHS Trust, Windmill Road, Headington, Oxford OX3 7LD, UK, phone: 01865 (7)38202,

e-mail: james.teh@ouh.nhs.uk
}

Authors' contribution:

A Study design · B Data collection · C Statistical analysis · D Data interpretation · E Manuscript preparation · F Literature search · G Funds collection 


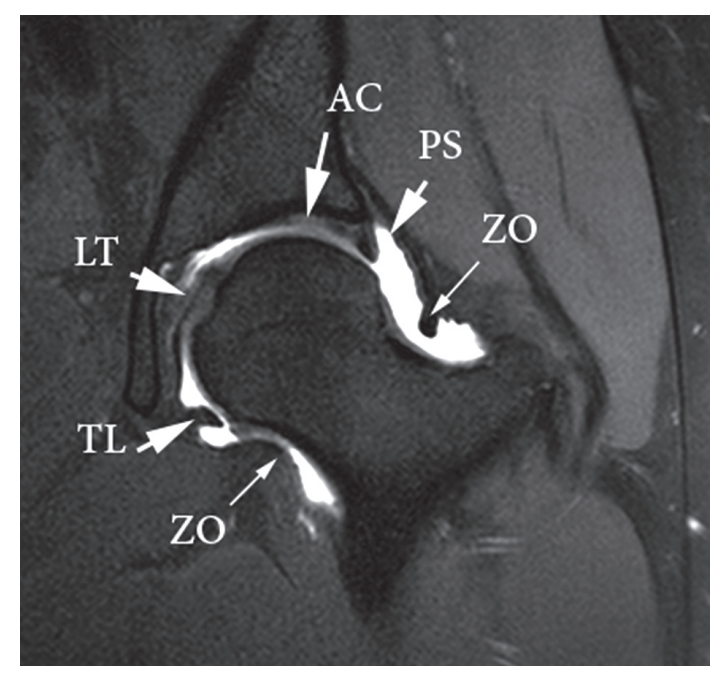

Figure 1. Normal anatomy of the hip on magnetic resonance arthrogram (MRA). Coronal T1-weighted, fat-saturated MRA image of the hip demonstrating the zona orbicularis (Z0), transverse ligament (TL), ligamentum teres (LT), and peri-labral sulcus (PS). The articular cartilage (AC) of the acetabulum is seen to be continuous with the labrum

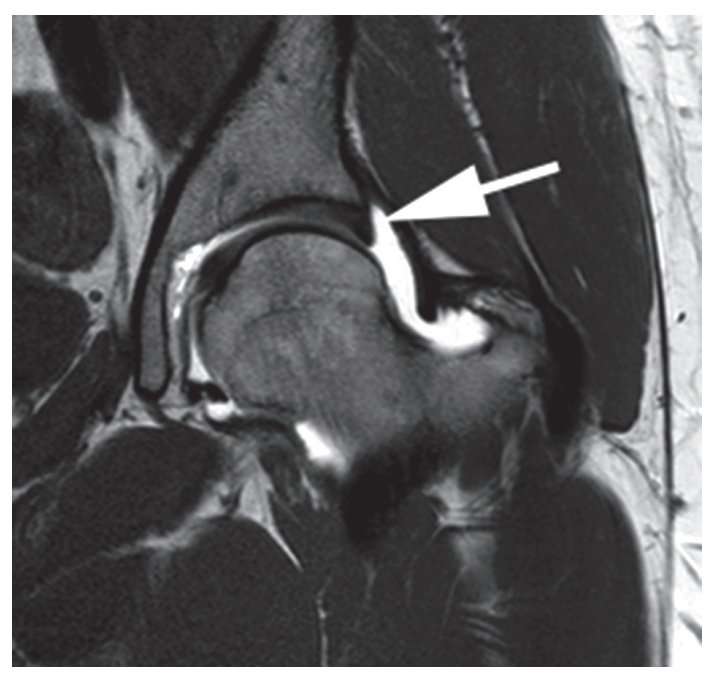

Figure 2. Normal anatomy of the hip on magnetic resonance arthrogram (MRA). Coronal T1W MRA image demonstrating homogeneous high $\mathrm{T} 1$ bone marrow signal. A normal low signal triangular acetabular labrum is seen (arrow)

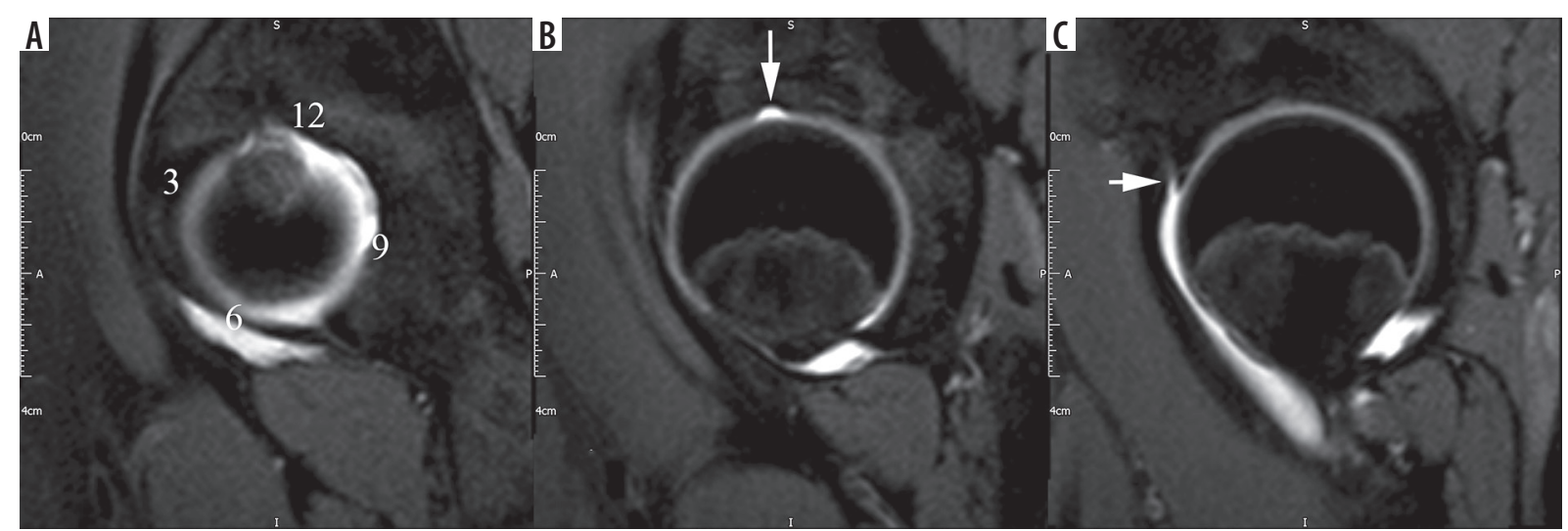

Figure 3. Normal anatomy of the hip on sagittal T1W fat-saturated magnetic resonance arthrogram images. A) The transverse acetabular ligament denotes the 6:00 position of the clock face, with the 3:00 position denoting the anterior midline aspect of the joint. B) Supraacetabular notch (arrow). C) Perilabral recess anterior to the intact labrum (short arrow)

content. The femoral neck and intertrochanteric region may have lower signal intensity on T1-weighted sequences due to the presence of red marrow and less fatty marrow content (Figures 1 and 2).

\section{Cartilage and labrum}

Articular hyaline cartilage is a complex combination of water, collagen, and proteoglycans, which help to distribute forces, absorb pressure, and enable gliding of the bony structures of the joint [4]. The entire femoral head is lined by articular hyaline cartilage, except for a small central depression known as the fovea capitis, where the ligamentum teres attaches [5]. The articular surface of the acetabulum is horseshoe-shaped and lined by hyaline cartilage, known as the lunate [6]. The central to inferior portion of the acetabulum is devoid of articular cartilage. The non-articular central depression of the acetabulum is known as the acetabular fossa, and is lined by synovium and filled with fibrofatty tissue/pulvinar [3]. A supraacetabular fossa is a normal variant of the acetabular roof seen in up to $10 \%$ of the population, consisting of a pseudodefect covered by cartilage or fibrous tissue at the 12 oclock position, and should not to be mistaken for an osteochondral defect $[3,4]$ (Figures 3 and 4). It is differentiated from a pathological lesion of the acetabulum by its smooth margins, absence of surrounding bone marrow oedema, and absence of cartilage irregularity. The stellate crease is a focal area devoid of hyaline cartilage, located at the inner margin of the acetabular cartilage, close to the superior aspect of the acetabular fossa (Figure 4). On MRI, normal articular hyaline cartilage has intermediate to high signal intensity on fluid-sensitive images. The evaluation of the articular cartilage of the hip joint is notoriously challenging due to the inherent curved morphology of the femoral head and acetabulum articular surfaces, the relatively thin articular hyaline cartilage measuring about 2 to $5 \mathrm{~mm}$ [7], and the close apposition of the articular surfaces [3]. 

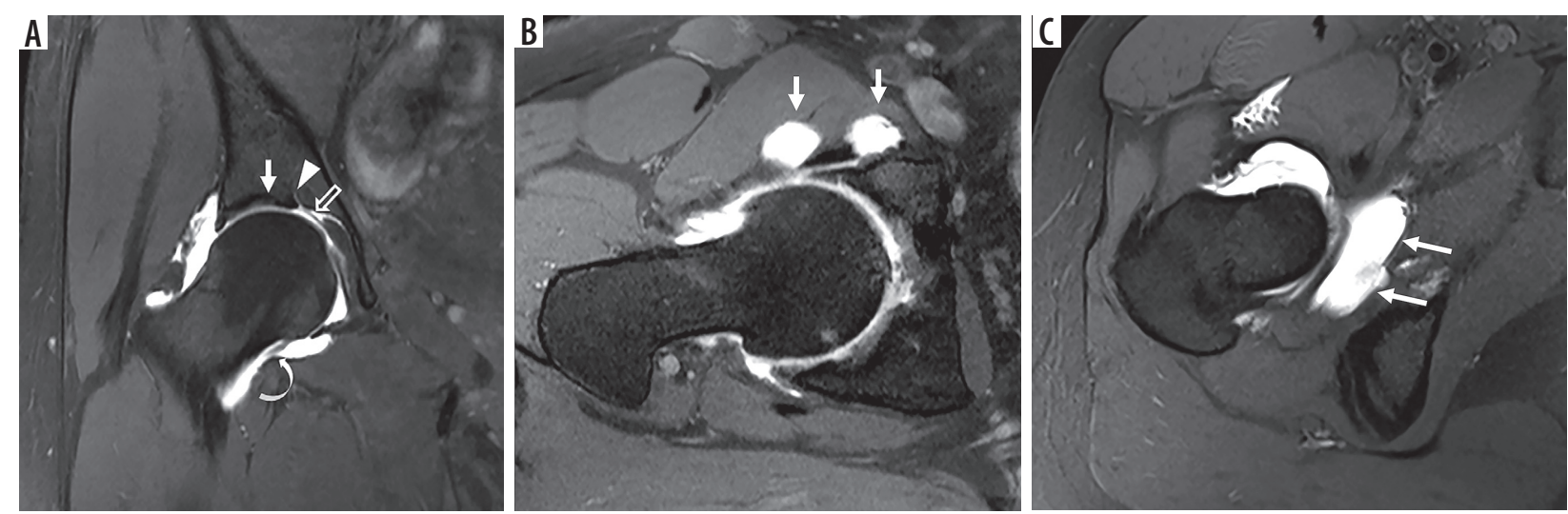

Figure 4. Normal bony and soft tissue variants on T1W fat-saturated magnetic resonance arthrogram images of the hip that can mimic pathology. A) Coronal image shows a supracetabular fossa located at 12:00 (arrow), medially positioned stellate crease (arrowhead), ligamentous plica extending towards the acetabular attachment of the ligamentum teres (open arrow), and a portion of the pectinofoveal fold (curved arrow). B) Axial oblique image shows normal communication between the hip joint and the iliopsoas bursa (arrows). C) Axial oblique image shows normal communication between the hip joint and the obturator externus bursa (arrows)

The acetabular labrum is a hypointense triangular fibrocartilaginous structure that is attached to the acetabular rim and blends with the transverse acetabular ligament at the margins of the acetabular notch inferiorly. The base of the labrum is immediately apposed to the outer margin of the acetabular cartilage, forming the chondrolabral junction [2]. The labrum is thought to have several important functions. It increases joint stability by increasing the acetabular depth and joint surface area. The labrum helps to more evenly distribute forces and enhance lubrication of the joint by forming a seal about the femoral head to maintain a trace of fluid under negative pressure within the joint space $[2,8]$. The location of intra-articular lesions may be described using the traditional clock-face method, which allocates the six oclock position as being the centre of the transverse acetabular ligament and the 12 oclock position as the superolateral aspect of the acetabulum (Figure 3). The three oclock position is usually assigned to the anterior midline and the nine oclock posterior midline location regardless of laterality. Another method divides the acetabulum into six geographic zones: anteroinferior as zone 1 , anterosuperior as zone 2 , superior as zone 3 , posterosuperior as zone 4 , posteroinferior as zone 5 , and acetabular fossa as zone 6 [9]. The six zones are applied to the femoral head in a corresponding manner.

Although the normal labrum is typically hypointense and triangular, there is variability in the morphological appearance and signal intensity of the acetabular labrum in asymptomatic individuals [10]. Intermediate to high signal intensity within the labral substance may be due to the presence of small intralabral fibrovascular bundles. Increased signal in the labrum on T1-weighted images may also be due to the magic angle effect [5]. There is a normal mildly hyperintense 1 to $2 \mathrm{~mm}$ transition zone at the chondrolabral junction [4]. Normal cartilage can 'undercut' the labrum at the chondrolabral junction, seen as a smooth focus of intermediate to high signal intensity (isointense to cartilage) interposed between the labrum and acetabular rim [3]. A sublabral sulcus is a fluid-filled cleft at the chondrolabral junction, typically located antero-inferiorly at the four oclock position [8], suggested to be a normal variant. A normal labro-ligamentous sulcus may be seen at the junction of the labrum and transverse acetabular ligament. Also, as the labrum degenerates with increasing age, it may become blunted and show signal alterations, or it may be absent.

Magnetic resonance arthrography (MRA) is the current gold standard for acetabular labral and hip hyaline articular cartilage assessment $[8,9]$, as fluid separates the internal structures and contrast extends into labral tears or chondral defects [5]. MRA has a higher diagnostic accuracy than non-contrast MRI for the detection of acetabular cartilage lesions at both $1.5 \mathrm{~T}$ and $3 \mathrm{~T}[11,12]$. However, despite the use of intra-articular contrast, the cartilage of the acetabulum and femoral head are often not delineated separately, and small lesions can be difficult to visualise. Hip MRA with leg traction has been shown to be a technically feasible and safe procedure that improves visualisation of the femoral and acetabular cartilage surfaces $[13,14]$. The leg traction distracts the hip joint allowing contrast to be interposed between the acetabular and femoral cartilage.

\section{Ligaments and capsule}

The ligamentum teres is an intra-articular ligament connecting the transverse acetabular ligament at the acetabular notch to the fovea capitis at the superomedial aspect of the femoral head [15]. The ligamentum teres transmits the foveal artery, which contributes only a little to the blood supply of the femoral head in adults [6] (Figure 1). On imaging, the ligamentum teres has a smooth contour and homogeneous low signal intensity on all pulse sequences $[15,16]$, or it may appear striated and have two to three bundles [17]. High signal from magic angle artifact may be seen at the femoral attachment. The ligamentum teres is well seen on coronal, axial, and axial oblique planes, but not on sagittal plane because of partial-volume averaging [15]. Con- 
genital absence of the ligament may occur, but its prevalence is uncertain.

The hip joint is lined by synovium along the inner surface of the joint capsule and reflected onto the labrum, transverse acetabular ligament, and ligamentum teres $[2,18]$. The joint capsule attaches proximally along the acetabular rim periosteum, usually near the base of the labrum [3]. This attachment creates a normal perilabral sulcus. The distal capsular attachment is at the femoral intertrochanteric line anteriorly, and higher on the femoral neck posteriorly. The joint capsule is reinforced by dense capsular ligaments that increase the stability of the hip joint. The capsular ligaments restrict hip joint motion beyond the normal range and protect the joint from edge loading. The capsular ligaments are the iliofemoral, ischiofemoral, and pubofemoral ligaments, named after the bones that they connect $[3,18]$ (Figure 5A). The iliofemoral ligament reinforces the anterior capsule, the ischiofemoral ligament reinforces the posterior capsule, and the relatively weaker pubofemoral ligament reinforces the anteroinferior capsule [2,3]. In addition, a deep layer of circular fibres at the femoral neck known as the zona orbicularis acts as a locking ring around the femoral neck to secure the femoral head within the acetabulum and resists joint distraction $[3,18]$. Synovial plicae are thin synovial folds within the joint, which are considered normal variants and are usually recognised in arthrography. This includes the ligamentous plica located within the acetabular fossa at the acetabular attachment of the ligamentum teres, labral plica at the inferomedial margin of the acetabular labrum, and the femoral neck plica, most commonly along the anterior joint capsule. The most commonly visualised plica is the pectinofoveal fold, which is a linear smooth or irregular thickening of the medial hip joint capsule, present in more than $95 \%$ of patients on MRA (Figure 4A). The iliopsoas bursa is located anterior to the hip joint about the iliopsoas tendon and has a normal communication with the hip in 10 to $15 \%$ of adult individuals [3] (Figure 4B). This is the largest bursa in the human body extending to the iliac brim. The obturator externus bursa, which also may be considered a joint recess, is a potential space located between the obturator externus tendon and the ischiofemoral capsular ligament at the posterior aspect of the hip joint capsule, and it should not be mistaken for capsular injury [19] (Figure 4C).

\section{Greater trochanter, tendons, and bursae}

The greater trochanter is the main attachment site for the hip abductors, comprising the gluteus medius and minimus muscles and external rotator tendons, which in turn includes the quadratus femoris, piriformis, obturator internus, obturator externus, and gemelli superior and inferior muscles. It has four bony facets: the anterior, lateral, supero-posterior, and posterior facets [20] (Figure 5B).

The gluteus minimus muscle consists of anterior and posterior parts and originates from the gluteal surface of the ilium, between the anterior and inferior gluteal lines. The tendon has medial and lateral bundles: The lateral bundle inserts at the anterior facet beneath the gluteus medius tendon, and the medial bundle is purely muscular and inserts anterosuperiorly onto the hip capsule. The fan-
A

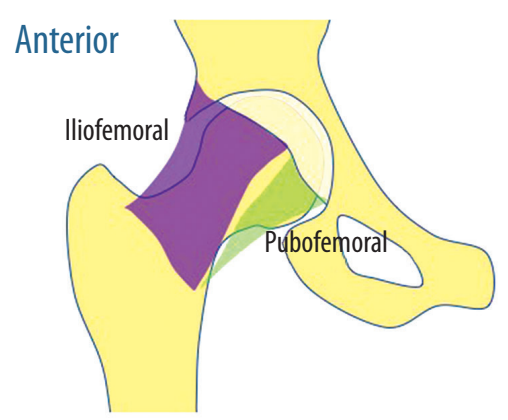

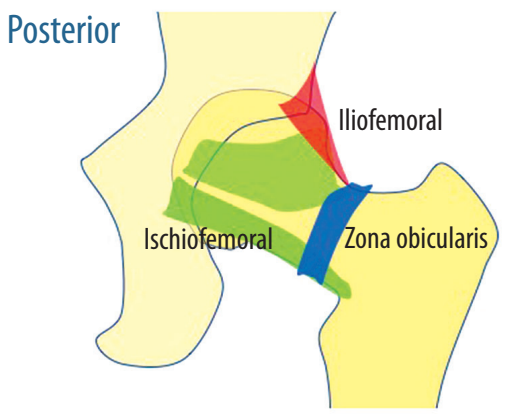
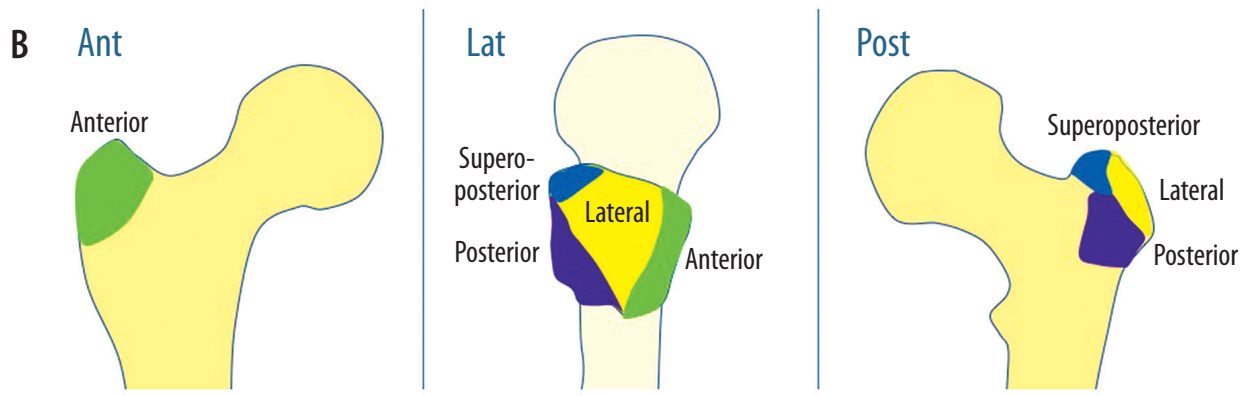

Figure 5. A) Schematic diagram of the anterior and posterior capsular ligaments of the hip joint. Anterior hip: iliofemoral (purple) and pubofemoral (green) ligaments. Posterior: ischiofemoral (green) and posterior iliofemoral (red) ligaments. The position of the zona orbicularis around the base of the femoral neck is demonstrated (blue). B) Schematic diagram of the facets of the greater trochanter. Ant - anterior hip, Lat - lateral hip, and Post - posterior hip 
shaped gluteus medius muscle arises from the iliac crest between the posterior and anterior gluteal lines and the gluteal aponeurosis and has anterior, central, and posterior parts. These give rise to three tendon bundles: the anterior part consists of muscle only and attaches onto the gluteus minimus tendon. The posterior and lateral bundles of the gluteus medius tendon insert at the supero-posterior and lateral facets, respectively [21]. These two gluteal muscles have similar features to the rotator cuff of the shoulder and are therefore considered the rotator cuff of the hip. The posterior facet of the greater trochanter has no tendon attachment. The trochanteric (subgluteus maximus) bursa covers the lateral and posterior facets and lies beneath the gluteus maximus muscle and iliotibial tract. The smaller subgluteus minimus and subgluteus medius bursae are located beneath the respective tendons but are usually not seen on MRI unless they are inflamed [21]. All the short external rotators insert at the inner medial margin of the greater trochanter, except for the quadratus femoris, which inserts at the posterior intertrochanteric ridge [21].

\section{Magnetic resonance imaging protocols}

MRI protocols for evaluation of the painful hip will vary between institutions, and according to the clinical scenario. The European Society of Skeletal Radiologists have suggested protocols for a standard MRI of the hip and a standard MRA of the hip [22]. As a general rule, highresolution, small field of view (FOV) $(15-20 \mathrm{~cm}$, with maximum slice thickness of $3.5 \mathrm{~mm}$ ) imaging of the affected hip should be obtained in all three planes. Highresolution flexible surface coils should be used.
Even if only one hip is symptomatic, there should be at least one wide FOV sequence that allows visualisation of the entire pelvis to enable exclusion of both intra- and extra-articular causes of hip pain (Tables 1 and 2). In the coronal plane the anterior to posterior acetabular columns should be included.

In the axial plane the anterior inferior iliac spine (AIIS) through lesser trochanter should be covered.

In the sagittal plane the medial acetabular wall through greater trochanter should be covered.

The axial-oblique plane should be obtained parallel to the femoral neck, and the FOV should cover the superior acetabular rim to the inferior acetabular rim. Radial slices are centred on the femoral head and are obtained perpendicular to the hip joint, providing true cross sections of the articular cartilage and acetabular labrum.

\section{Osseous lesions}

\section{Occult hip fractures}

Most hip fractures are readily diagnosed on radiographs $[6,23]$. In ambiguous cases, MRI has an established role for detection of radiographically occult fractures [6]. On MRI, a non-displaced fracture is seen as a hypointense line, which is best depicted on T1-weighted sequences, surrounded by hyperintense bone marrow oedema on fluidsensitive sequences. MRI also allows delineation of associated soft tissue injury. Occult fractures may result from either acute trauma or prolonged repetitive microtrauma.

Fractures that result from the cumulative effect of repetitive microtrauma are termed stress fractures [23], and they

Table 1. Standard hip magnetic resonance imaging protocol, adapted from European Society of Musculoskeletal Radiology guidelines [22]

\begin{tabular}{|l|c|c|c|c|c|}
\hline Parameter & FOV (max) & Slice (max) & TE & TR & Matrix (min) \\
\hline Cor STIR (whole pelvis) & $38-40 \mathrm{~cm}$ & $6 \mathrm{~mm}$ & $40-60$ & 1800 & $256 \times 256$ \\
\hline Cor T1 & $16 \mathrm{~cm}$ & $3.5 \mathrm{~mm}$ & $\min$ & 400 & $256 \times 256$ \\
\hline Cor PD/T2 FS & $16 \mathrm{~cm}$ & $3.5 \mathrm{~mm}$ & $40-100$ & 2000 & $256 \times 256$ \\
\hline Sag PD/T2 FS & $16 \mathrm{~cm}$ & $3.5 \mathrm{~mm}$ & $40-60$ & 2000 & $256 \times 256$ \\
\hline Ax-oblique PD FS & $16 \mathrm{~cm}$ & $3.5 \mathrm{~mm}$ & $40-60$ & 2000 & $256 \times 256$ \\
\hline
\end{tabular}

FOV - field of view, FS - fat-saturated, STIR - short-tau inversion recovery, PD - proton density

Table 2. Standard hip magnetic resonance arthrogram protocol, adapted from European Society of Musculoskeletal Radiology guidelines [22]

\begin{tabular}{|l|c|c|c|c|c|}
\hline Parameter & FOV (max) & Slice $(\mathrm{max})$ & TE & TR & Matrix (min) \\
\hline Cor STIR (whole pelvis) & $38-40 \mathrm{~cm}$ & $6 \mathrm{~mm}$ & $40-60$ & 1800 & $256 \times 256$ \\
\hline Ax T1 & $16 \mathrm{~cm}$ & $3.5 \mathrm{~mm}$ & $\min$ & 400 & $256 \times 256$ \\
\hline Cor T1 FS & $16 \mathrm{~cm}$ & $3.5 \mathrm{~mm}$ & $\min$ & 400 & $256 \times 256$ \\
\hline Oblique T1 FS & $16 \mathrm{~cm}$ & $3.5 \mathrm{~mm}$ & $\min$ & 400 & $256 \times 256$ \\
\hline Sag T1 FS & $16 \mathrm{~cm}$ & $3.5 \mathrm{~mm}$ & $\min$ & 400 & $256 \times 256$ \\
\hline Sag or oblique PD FS & $16 \mathrm{~cm}$ & $3.5 \mathrm{~mm}$ & $40-60$ & 2000 & $256 \times 256$ \\
\hline Radial T1 \pm FS (optional) & $16 \mathrm{~cm}$ & $3.5 \mathrm{~mm}$ & $\min$ & 400 & $256 \times 256$ \\
\hline
\end{tabular}

FOV - field of view, FS - fat-saturated, STIR - short-tau inversion recovery, PD - proton density 
tend to be difficult to detect radiographically because they are often non-displaced. However, if continued stresses are placed upon the affected bone, the fracture may become displaced [6]. Stress fractures are characterised as either fatigue or insufficiency type. Fatigue fractures occur in normal bones undergoing excessive loading, while insufficiency fractures occur in weakened bone undergoing normal loading [6]. MRI is the most sensitive and accurate imaging modality for detecting and grading stress injuries $[2,23]$ (Table 3).

Femoral neck stress fractures are classified as compression sided or tension sided [24]. Compression-sided injuries occur at the inferomedial femoral neck and have low risk of displacement, and they can typically be managed conservatively (Figure 6). Tension-sided injuries occur at the superolateral femoral neck and have a greater risk for displacement, and therefore usually necessitate surgery. MRI is also used to detect radiographically occult intertrochanteric or cervical fracture extension in the setting of known greater trochanteric fractures [25]. If there is no extension, then there is the option of non-operative management. Isolated lesser trochanteric fractures in adults are usually pathological avulsions, and work up for metastatic disease should be initiated.

In recent years there has been increased recognition of subchondral insufficiency fractures (SIF) of the weightbearing portion of the femoral head as a distinct entity from osteonecrosis (ON). SIF tend to occur in older, obese osteoporotic patients, more often in women than men. Differentiating SIF from ON may be difficult on MRI, but with
SIF, an irregular serpiginous low T2 signal subchondral fracture line/band parallel to the articular surface of the femoral head is seen [26]. Alternatively, there is condensation of the subchondral bone with surrounding oedema. This band lesion is the main finding, which histopathologically corresponds to a fracture line with associated repair tissue. In ON, the low-intensity band is smooth, concave to the articular surface, circumscribes all of the necrotic segments, and has a geographical border. SIF tends to be unilateral, whereas $\mathrm{ON}$ is often bilateral. With progression of ON there may be collapse of the femoral head, making differentiation from collapse due to SIF impossible (Figure 7).

\section{Osteonecrosis}

Osteonecrosis is a pathologic process that results from interruption of the vascular supply to a bone. The femoral head is particularly vulnerable to developing $\mathrm{ON}$ due to the nature of its blood supply [6]. The more common risk factors for developing $\mathrm{ON}$ are chronic corticosteroid use and alcohol abuse [6]. Therefore, the threshold for imaging in these patients who have hip pain should be low because they are predisposed to ON [27]. ON progresses from radiologically occult disease to articular collapse and eventually secondary osteoarthritis (OA). Therefore, early diagnosis of $\mathrm{ON}$ is essential because it allows earlier intervention to prevent irreversible joint damage. MRI is considered the most sensitive imaging modality in the early detection of $\mathrm{ON}$ and plays an important role in its staging [6,28]. On T1-weighted images,

Table 3. Grading of stress fractures

\begin{tabular}{|l|l|}
\hline Stress fracture & Magnetic resonance imaging features \\
\hline Grade 1 & Subtle periosteal soft tissue oedema on fluid-sensitive sequence \\
\hline Grade 2 & Mild subcortical bone marrow oedema and periosteal soft tissue oedema on fluid-sensitive sequences \\
\hline Grade 3 & Extensive bone marrow oedema and periosteal soft tissue oedema on both T1-weighted and fluid-sensitive sequences \\
\hline Grade 4 & Discrete hypointense fracture line on all sequences \\
\hline
\end{tabular}
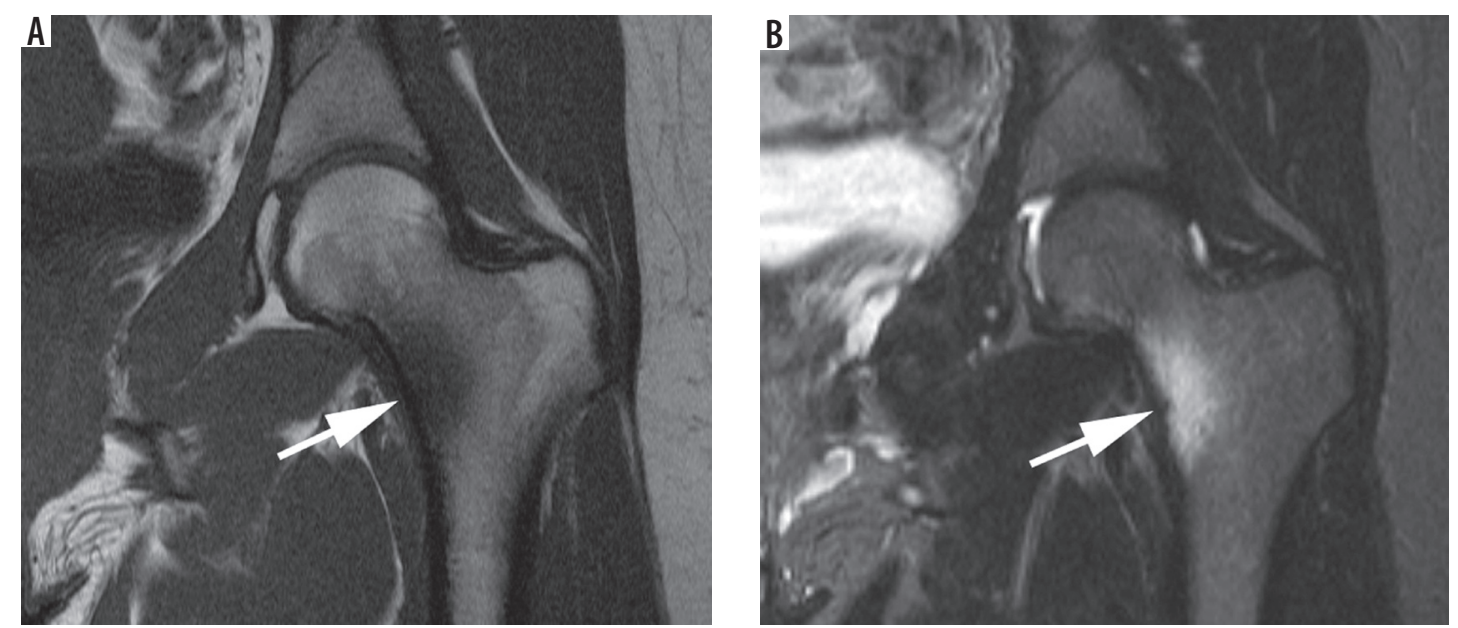

Figure 6. Compression type stress fracture at the medial aspect of the left femoral neck base in a 21-year-old runner. A) Coronal T1W magnetic resonance (MR) image demonstrating low T1 signal (arrow) in the medial femoral neck. B) Coronal short-tau inversion recovery. MR image demonstrating an incomplete fracture line perpendicular to the cortex with surrounding high signal bone marrow oedema (arrow) 

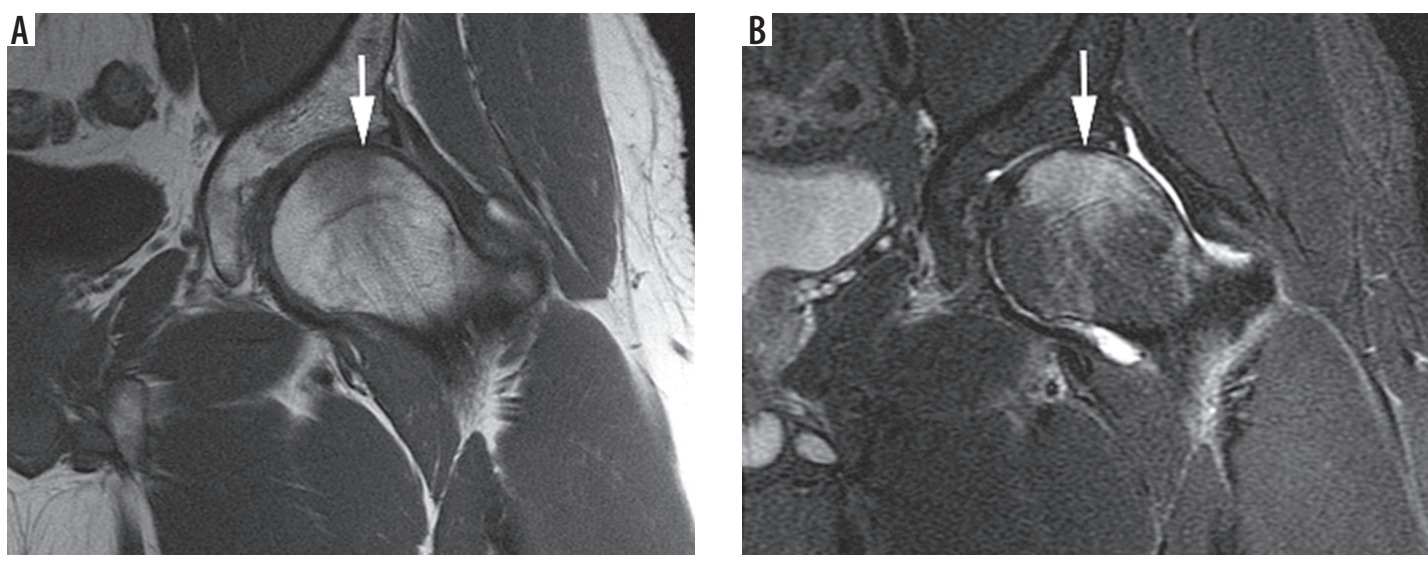

Figure 7. Subchondral insufficiency fracture of the left femoral head. A) Coronal T1W magnetic resonance (MR) image demonstrating a band of subchondral low signal intensity in the weight-bearing portion of the femoral head consistent with a fracture line (arrow). B) Coronal short-tau inversion recovery MR image demonstrating subchondral low signal condensation/fracture line (arrow) with surrounding high signal intensity bone marrow oedema

the area of necrosis is usually seen as a geographic region of fat signal intensity surrounded by a low signal intensity rim. The fatty marrow within the necrotic area remains hyperintense on T1-weighted images because both viable and devitalised adipose tissues have identical intrinsic MRI signal intensity $[28,29]$. Less commonly, the signal intensity in the necrotic area may reflect haemorrhage, cyst-like change, or fibrous tissue [28]. On T2-weighted images, the doubleline sign is virtually pathognomonic of $\mathrm{ON}$ and is present in up to $80 \%$ of cases [6]. The sign describes two immediately apposed lines: an inner line of high signal on the necrotic side representing reparative granulation tissue of the reactive interface, and an outer line of low signal on the healthy side representing sclerosis to wall off the osteonecrotic process $[6,28]$ (Figure 8). The double-line sign may also partially result from chemical-shift misregistration artifact [28]. Intravenous contrast is not necessary but if administered, the devitalised tissue will be non-enhancing with a peripheral rim of enhancement at the granulation tissue [28]. Quantifying the percentage of weight-bearing femoral cortex involved in ON using MRI has been found to be a reliable parameter in determining outcome. ON with less than $25 \%$ involvement of the femoral head appears to benefit greatly from core decompression, while ON with more than $50 \%$ involvement has a very poor prognosis despite core decompression [6]. As the condition is bilateral in up to $40 \%$ of cases, simultaneous imaging of both hips is strongly recommended [6]. There are several different classifications of ON [30,31]. The most widely used classification system is the Ficat and Arlet classification, which utilises a combination of radiographs, MRI, and clinical features to stage ON of the femoral head [30,32] (Table 4). The main differentials for ON are transient osteoporosis of the hip (TOH) and SIF.

The femoral head consists of medial, central, and lateral pillars. In Legg-Calve-Perthes disease and ON the early appearance of lateral pillar enhancement following intravenous gadolinium is indicative of uncomplicated revascularisation of the femoral head associated with a good prognosis [33,34] (Figure 9). Conversely, enhancement of the metaphyseal region is reported in the literature as having a predictive value for a poor prognosis.

Diffusion-weighted imaging (DWI) allows identification of subtle interstitial movement of water, and thus enables an indirect evaluation of the tissue structure in bone marrow. In normal marrow, the trabeculae and fat cells result in structural complexity, but in $\mathrm{ON}$ when blood cells, osteocytes, and marrow fat cells die, the components of the matrix break down, allowing water diffusion in bone marrow to become more active, leading to increased diffusion and an increased apparent diffusion coefficient (ADC). On DWI increased metaphyseal diffusivity (ADC) correlates well with absent lateral pillar enhancement on contrast enhanced MRI, which may therefore indicate an unfavourable prognosis $[35,36]$. Low or normal diffusivity therefore correlates with a better prognosis (Figure 10). There has also been correlation between the stage of Legg-Calve-Perthes disease and the ADC value [33]. In adults, however, it remains controversial how much value DWI adds over conventional MRI because staging, prognosis, and treatment planning of $\mathrm{ON}$ can be achieved without DWI.

\section{Transient osteoporosis}

The hip joint is the most common location of transient osteoporosis, which is also referred to as transient bone marrow oedema syndrome [37]. $\mathrm{TOH}$ is a self-limiting condition that resolves within 6 to 12 months of conservative management with reduced weight bearing. Although $\mathrm{TOH}$ is classically associated with women in the third trimester of pregnancy, it is actually more commonly seen in middle-aged men [6], but the pathogenesis remains unclear [37]. MRI is the imaging modality of choice, showing diffuse bone marrow oedema with a wide zone of transition in the femoral head and neck on fluid-sensitive sequences and diffuse enhancement after administration of intravenous contrast. A reactive joint effusion is a frequent finding. $\mathrm{TOH}$ is a diagnosis of exclusion, but the 

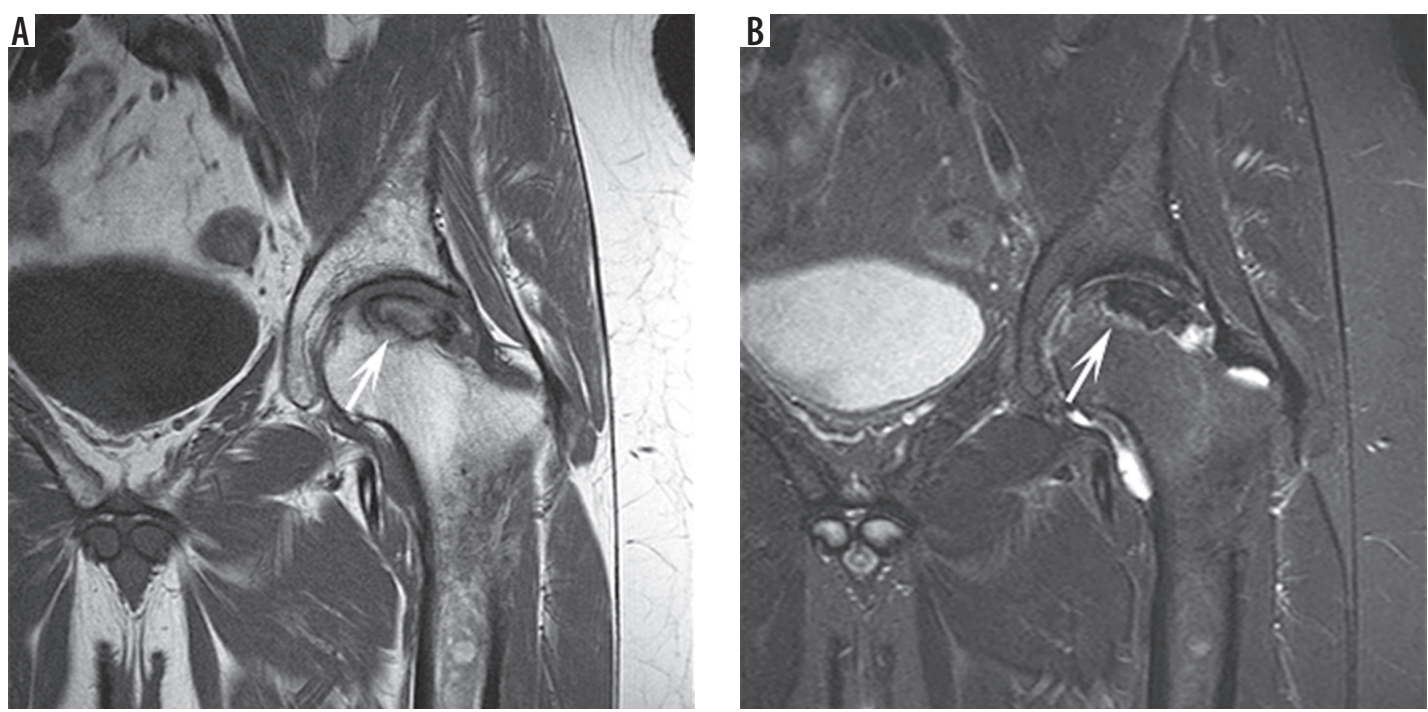

Figure 8. Osteonecrosis of the femoral head. A) Coronal T1W magnetic resonance (MR) image demonstrating a low/intermediate signal geographic lesion in the subchondral region of the proximal femoral head surrounded by a low signal intensity rim (long arrow). B) Coronal short-tau inversion recovery MR image demonstrating a geographic lesion of low signal intensity in the proximal aspect of the femoral head with an inner line of high signal on the necrotic side representing reparative granulation tissue of the reactive interface and an outer line of low signal representing sclerosis, described as the "double line" sign (long arrow)

Table 4. Ficat and Arlet classification [32] (modified) for osteonecrosis

\begin{tabular}{|l|l|}
\hline Stage & Findings \\
\hline 0 & $\begin{array}{l}\text { Radiography: normal } \\
\text { MRl: } \text { normal } \\
\text { Clinical symptoms: none }\end{array}$ \\
\hline 1 & $\begin{array}{l}\text { Radiography: normal or minor osteopaenia } \\
\text { MRl: bone marrow oedema } \\
\text { Bone scan: increased tracer uptake } \\
\text { Clinical symptoms: groin/hip pain }\end{array}$ \\
\hline 2 & $\begin{array}{l}\text { Radiography: mixed osteopaenia and/or sclerosis and/or } \\
\text { subchondral cyst-like changes, without any subchondral lucency } \\
\text { MRl: geographical signal change } \\
\text { Bone scan: increased uptake } \\
\text { Clinical symptoms: pain and stiffness }\end{array}$ \\
\hline 3 & $\begin{array}{l}\text { Radiography: crescent sign (subchondral lucency/fracture) } \\
\text { and eventual cortical collapse } \\
\text { MRl: femoral head collapse, joint preserved } \\
\text { Clinical symptoms: pain and stiffness, limp }\end{array}$ \\
\hline 4 & $\begin{array}{l}\text { Radiography: end-stage with evidence of secondary OA } \\
\text { MRl: femoral head collapse and OA } \\
\text { Clinical symptoms: pain and limp }\end{array}$ \\
\hline
\end{tabular}

MRI - magnetic resonance imaging, $\mathrm{OA}$ - osteoarthritis

main diagnostic dilemma lies with $\mathrm{ON}$, because a diffuse oedema pattern is common to both entities. However, the absence of focal defects and subchondral changes is highly suggestive of TOH $[6,37,38]$ (Figure 11).

\section{Intra-articular pathology}

\section{Ligamentum teres femoris injuries}

Ligamentum teres femoris injuries include traumatic and degenerative tears, and avulsion fractures at the fovea

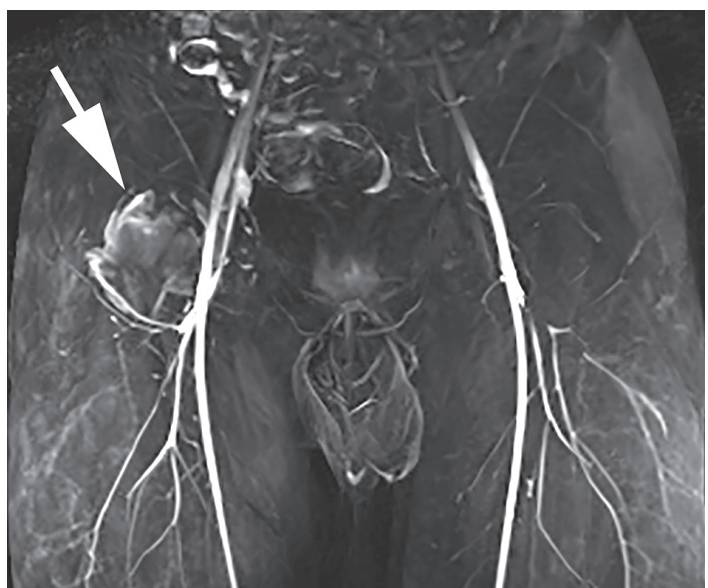

Figure 9. Osteonecrosis of the right femoral head in a 21-year-old male treated with steroids for acute lymphoblastic leukaemia. Perfusion T1W fat-saturated post-contrast coronal magnetic resonance angiogram image demonstrating increased uptake of contrast in the lateral pillar (arrow), suggesting a good prognosis

capitis femoris, and it may account for $4-15 \%$ of sportsrelated injuries [16]. Patients with a torn ligamentum teres develop microinstability, which can be aggravated by sporting activity leading to damage of the cartilage or labrum. Tears of the ligamentum teres have been classified into three types on the basis of arthroscopic findings: complete rupture (type I), partial tearing (type II), and degenerative ligament tear (type III) [39]. Ruptures tend to occur at the foveal attachment and are typically associated with hip dislocation, but they may occur in the setting of stresses on the joint in flexion-adduction or twisting injuries with hyper-abduction. Degenerative tears occur in the context of OA. When the ligamentum teres is injured there is loss of the normal low T2 signal on fluid-sensitive sequences, indicating oedema and/or haemorrhage. The ligament may be thickened with loss of the fascicular 


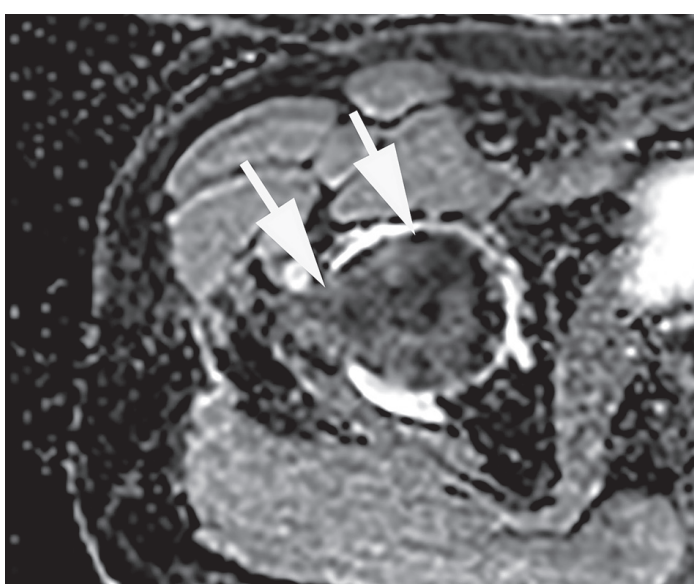

Figure 10. Osteonecrosis in a 21-year-old male treated with steroids for acute lymphoblastic leukaemia (the same patient as in Figure 8). Diffusion-weighted magnetic resonance imaging of the right hip demonstrating no restricted diffusion in the proximal femur on the axial apparent diffusion coefficient map (arrows) indicating a good prognosis

structure indicating an interstitial tear (Figure 12). Secondary signs of injury include oedema in the acetabular fossa, synovitis, and hip joint effusion [16].

\section{Lesions of the acetabular labrum}

Acetabular labral injuries are the consequences of three main pathomechanisms which are not mutually exclusive: joint instability due to hip dysplasia, FAI, and levering out (Figure 13). Labral injuries are therefore encountered in a wide range of conditions including trauma, FAI, hip dysplasia, Legg-Calve-Perthes, and slipped capital femoral epiphysis (SCFE). The acetabular labrum is relatively avascular with limited healing potential [2]. Labral tears may result in mechanical hip pain, instability, giving way of the joint, clicking, and locking [6]. However, it is important to remember that labral tears are also highly prevalent in the asymptomatic population [1]. There is a highly significant association between the presence of labral lesions and adjacent cartilage damage, and this predisposes to development of early OA of the hip [40].

There are several surgical and MRI classifications of labral tears, but there is a weak agreement between these classifications [41-43]. The focus should therefore be on an accurate descriptive report detailing the tear position, size, and associated cartilage and osseous changes. Labral tears may be subdivided into intrasubstance and intersubstance tears, or a combination of both. Intrasubstance tears involve the labral body, while intersubstance tears involve the labral base at the chondrolabral junction. Intersubstance tears start as chondrolabral separation and progress to partial then complete detachments of the labrum from the acetabular rim [7] (Figures 14 and 15). Labral detachments are more common than intrasubstance labral tears [5]. Labral detachment is a characteristic finding in cam FAI, while intrasubstance labral tears are commonly seen in pincer FAI and hip dysplasia (Figure 16).

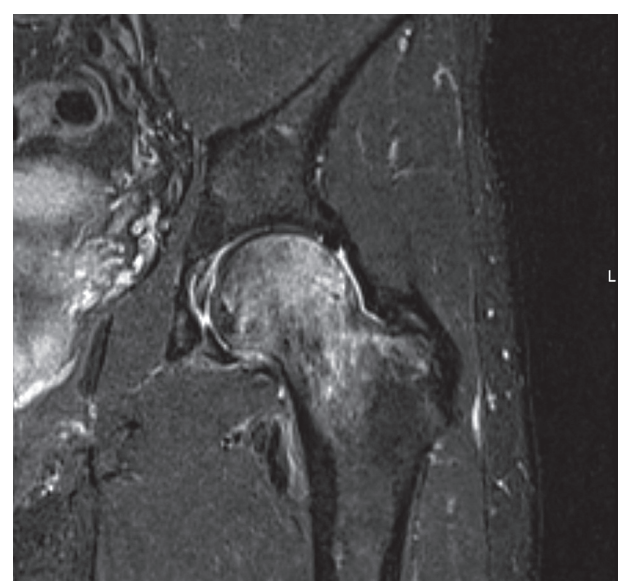

Figure 11. Transient osteoporosis. Coronal short-tau inversion recovery magnetic resonance image of the left hip demonstrating extensive bone marrow oedema in the left femoral head and neck without discrete fracture line or additional subchondral abnormality

On MRI, labral tears and detachments typically appear as linear hyperintense signal intensity on fluid-sensitive sequences. MRA is the imaging technique of choice for evaluating the labrum and is most consistent in detecting labral tears and detachments $[6,11,44]$. On MRA, intrasubstance tears are diagnosed by the presence of intrasubstance contrast material (Figure 17). Contrast at the acetabular-labral interface represents the typical appearance of labral detachment on MRA [6] (Figure 15B).

Labral tears and detachments occur in the anterosuperior and anterior locations in up to $92 \%$ of cases $[2,3]$. Therefore, high signal intensity seen at the anterosuperior or anterior labrum is statistically more likely to represent a tear or detachment rather than a normal sulcus [3]. On the other hand, increased signal intensity at the posteroinferior chondrolabral junction is more likely to be a sulcus because lesions are rare in this location [3]. Anterosuperior and anterior labral lesions are best seen on sagittal

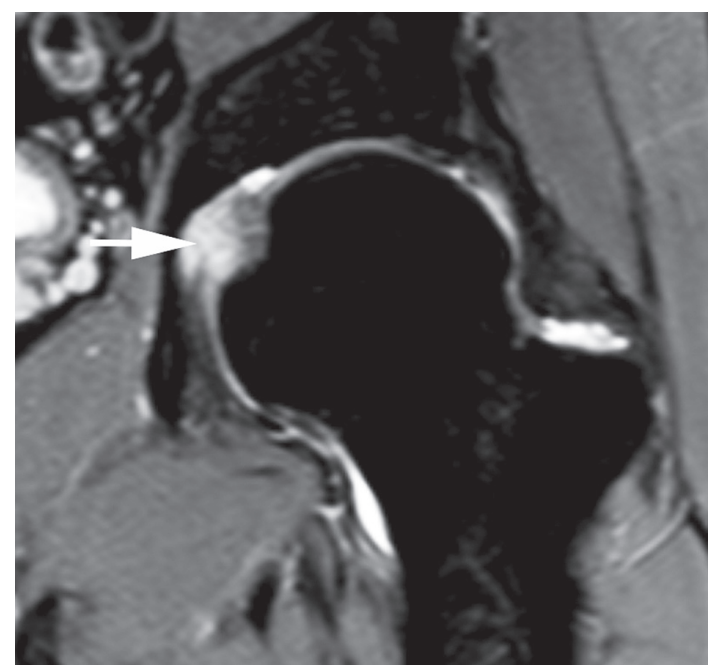

Figure 12. Ligamentum teres tear. Coronal proton density-weighted fatsaturated magnetic resonance image demonstrating blurring, swelling, and oedema of the ligamentum teres consistent with tearing (arrow) 

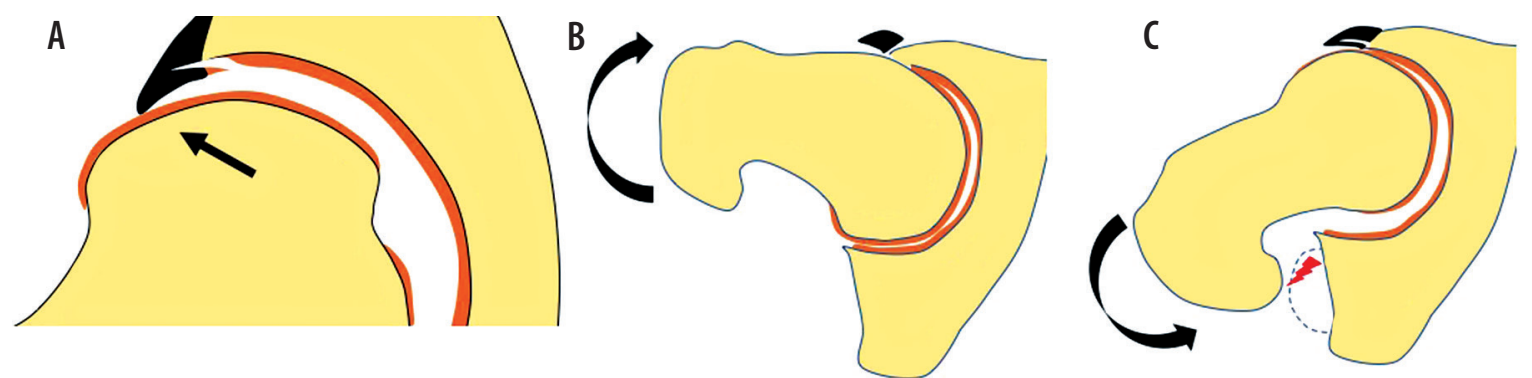

Figure 13. Pathomechanisms of acetabular labral tears. A) Hip dysplasia: subluxation of the femoral head results in "inside-out" tears the hypertrophied labrum from the acetabular rim together with a sleeve of cartilage. Typically, this results in an intrasubstance labral tear. B) Impingement: dynamic abutment of the femur against the acetabular rim as seen with femoroacetabular impingement. Typically, this is results in an intersubstance tear. C) Levering out: femoral torsional abnormality resulting in impingement against the ischium. Typically, this results in an intrasubstance tear

and axial oblique images whereas superior labral lesions are best identified on coronal images [2].

Paralabral cysts are secondary findings to labral tears and detachment, most commonly on the capsular surface of the labrum [4]. Paralabral cysts are formed when sy-

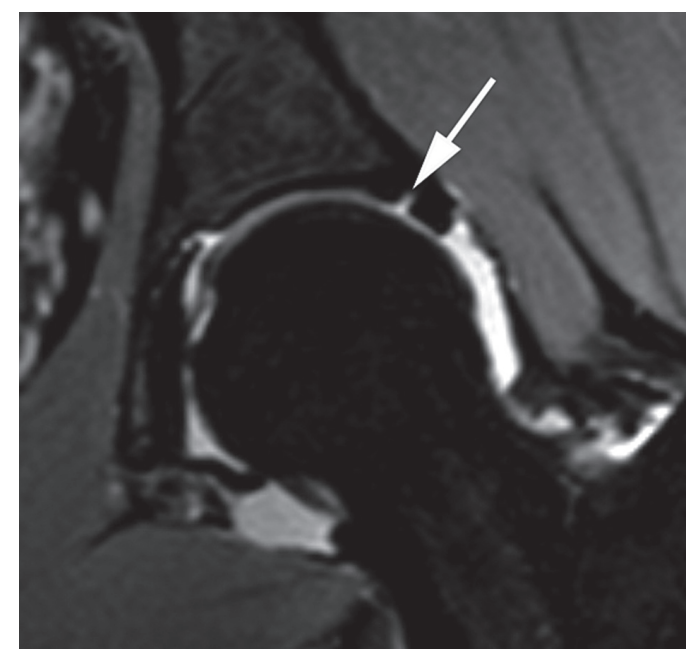

Figure 14. Partial intersubstance tear. Coronal T1W fat-saturated magnetic resonance arthrogram image of the hip demonstrating a contrast-filled partial intersubstance tear (arrow) at the chondrolabral junction
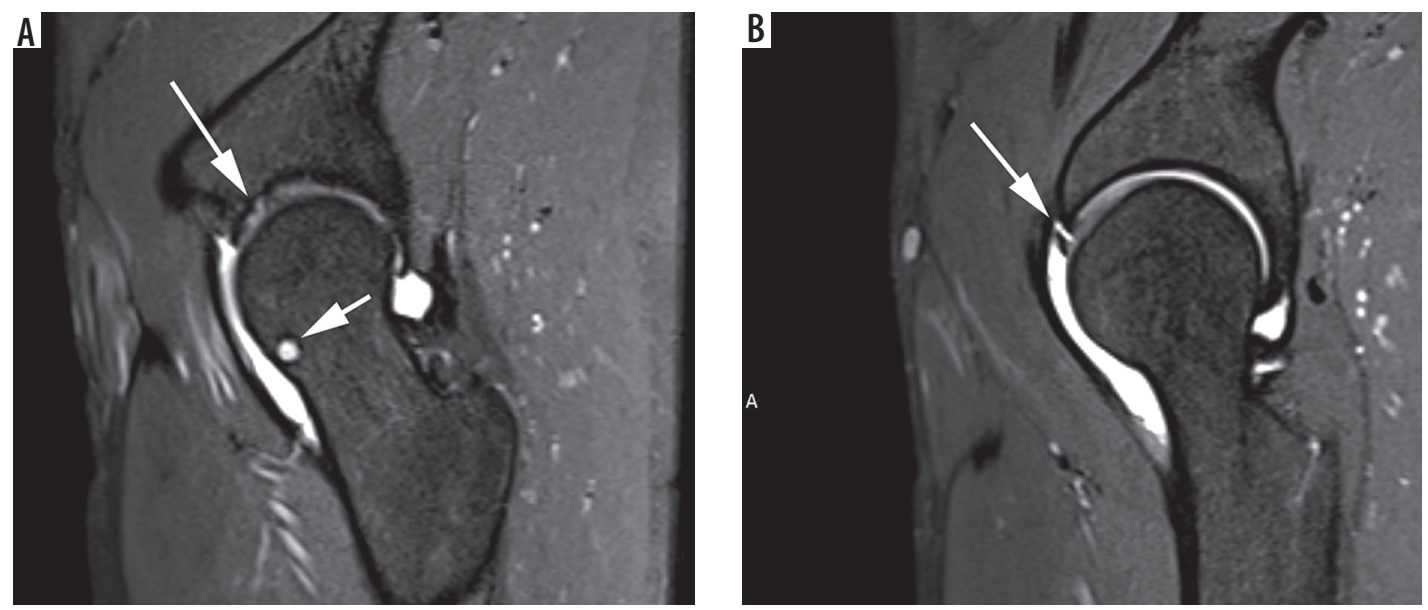

Figure 15. Articular cartilage loss, herniation pit and chondrolabral separation on sagittal T1W fat-saturated magnetic resonance arthrogram images. A) Note partial-thickness articular cartilage loss at the acetabular margin (long arrow) and a synovial herniation pit (short arrow). B) There is an intersubstance anterior chondrolabral junction tear (arrow) 


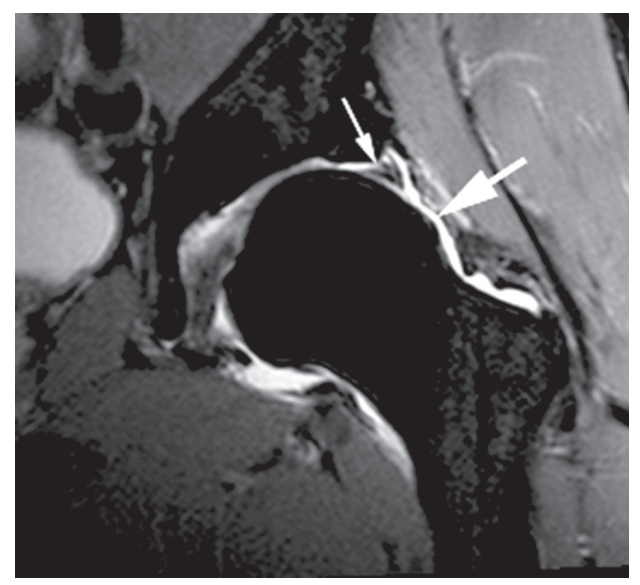

Figure 16. Cam morphology and acetabular labral tear. Coronal T1W fatsaturated magnetic resonance arthrogram image showing a bone protuberance at the lateral aspect of the femoral head and neck junction consistent with cam morphology (large arrow), and a chondrolabral junction intersubstance tear (small arrow). Note thinning of the articular cartilage at the acetabular margin

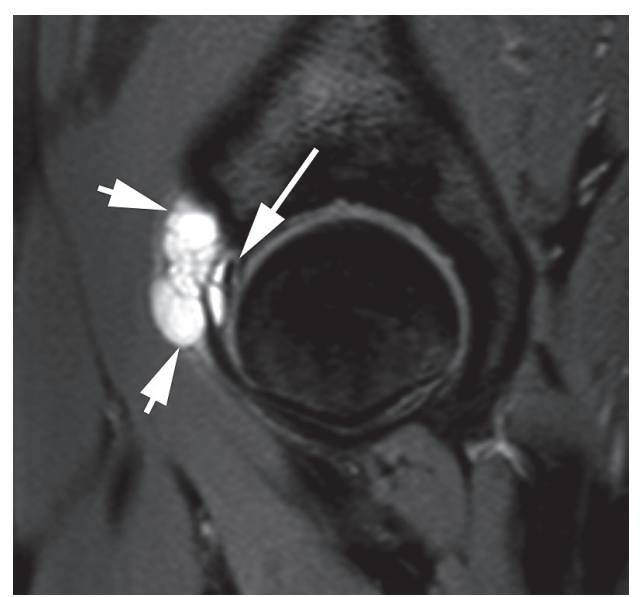

Figure 18. Complex acetabular labral tear with paralabral cyst. Sagittal proton density fat-saturated magnetic resonance image demonstrating a lobulated multiloculated fluid signal intensity lesion at the anterior aspect of the hip consistent with a paralabral cyst (short arrows) in association with a complex labral tear (long arrow)

is considered the imaging technique of choice $[13,14]$. On MRI, normal articular hyaline cartilage has intermediate to intermediate-high signal intensity. Heterogeneous signal in the articular cartilage probably represents the earliest stage of degeneration or chondromalacia. Chondral lesions are described as thinning, fraying or fissuring, partial or full thickness defects, and fissures, flaps, or delamination [9,45] (Figure 20).

Secondary findings associated with chondral lesions include subchondral marrow oedema-like signal and subcortical/subchondral cyst-like changes, best seen on fluidsensitive sequences (Figure 19).

\section{Femoroacetabular impingement}

Femoroacetabular impingement is a dynamic phenomenon that results from conflict between the proximal

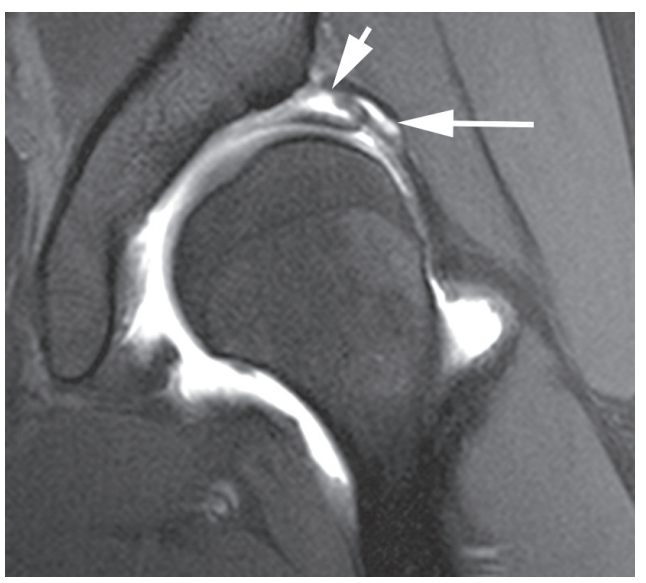

Figure 17. Developmental hip dysplasia (DDH) with acetabular labral tear. T1W fat-saturated magnetic resonance arthrogram image demonstrating DDH with lateral uncovering of the femoral head. There is an "inside out" intrasubstance labral tear (short arrow), and a small paralabral cyst that has filled with contrast (long arrow)

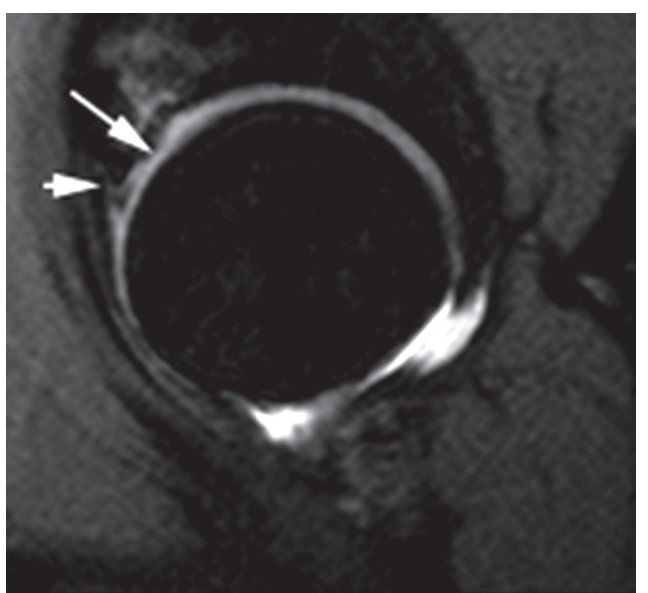

Figure 19. Cartilage loss and labral tear. Sagittal proton density fat-saturated magnetic resonance image demonstrating an anterior superior labral tear (short arrow) with > 50\% articular cartilage loss at the acetabular margin (long arrow) and subjacent high signal consistent with subarticular stress change in the acetabulum

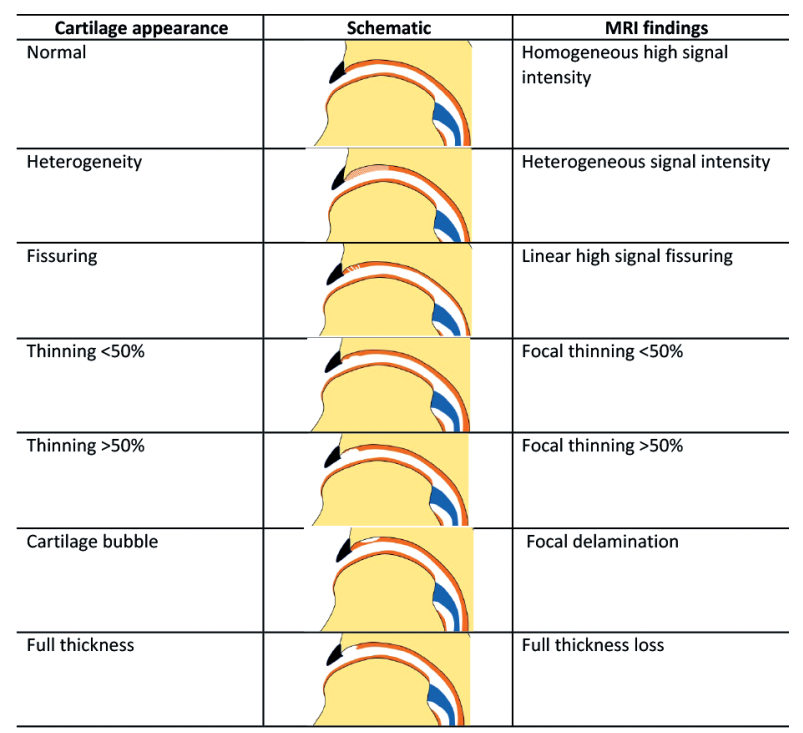

Figure 20. Schematic description of hyaline cartilage lesions 
femur and the acetabular rim during hip motion $[4,46]$. It is caused by abnormal bony morphology, especially when the joint is subjected to exertional forces and a range of motion beyond normal physiological limits $[4,46]$. The bony abnormality may be at the femoral head-neck junction (cam morphology), acetabular rim (pincer morphology), or a combination of both (mixed). A synovial herniation pit, also known as "Pitt's pit", or fibrocystic change is a well-circumscribed round or oval high T2 signal defect located in the subchondral or subcortical region of the anterosuperior femoral neck [47] (Figure 15A). Imaging studies demonstrate a significant predominance of mixed FAI $[2,4]$. The repetitive microtrauma from the bony conflict leads to labral and cartilage damage, and FAI is recognised as an important cause of premature OA [6].

Cam and pincer morphology as well as the intra-articular damage from the impingement are well depicted on MRI. Due to the paradigm shift towards joint-preserving procedures of the hip, MRI plays an increasing role in preoperative assessment for cam and pincer osseous deformities and detection of early intra-articular changes before irreversible cartilage damage occurs. The predominant findings in cam, pincer, and mixed types of FAI are described in Table 5.

\section{Cam morphology}

Cam morphology is a protuberant femoral headneck junction, which may be focal or generalised. There is a strong association between sporting activity and the development of FAI. A meta-analysis has shown that young male athletes between the ages of 12 and 21 years are two to eight times more likely to develop a cam-type deformity than controls [48]. It is thought that high-impact sports during skeletal maturation increases the risk of developing cam morphology as an adaptive response at the femoral physis from increased mechanical stimuli. SCFE, Legg-Calve-Perthes and post-traumatic conditions can also alter the sphericity of the femoral head. It is important to note that cam morphology is also common in asymptomatic individuals.

Table 5. Predominant imaging findings in femoroacetabular impingement

\begin{tabular}{|l|c|c|c|}
\hline Parameter & Cam & Pincer & Mixed \\
\hline Age & Young & Middle-aged & Young to middle-aged \\
\hline Sex & $\mathrm{M}$ & $\mathrm{F}$ & $\mathrm{M}+\mathrm{F}$ \\
\hline Femoral bump & + & - & + \\
\hline Cartilage fissure & + & + & + \\
\hline Cartilage delamination & + & - & + \\
\hline Labral tear & + & + & + \\
\hline Labral detachment & + & - & \pm \\
\hline Os acetabulum & \pm & + & \pm \\
\hline Synovial herniation pit & + & \pm & \pm \\
\hline
\end{tabular}

The osseous prominence can occur anywhere along the femoral head-neck junction but is most commonly located at the anterosuperior aspect, known as the pistol-grip deformity. This is best seen on the MRI in axial oblique plane and may be quantified in a variety of ways. The alpha angle is the most common method used to quantify the degree of asphericity of the femoral head with a threshold of above 55 to 60 degrees being abnormal $[2,49]$. The alpha angle is evaluated on the axial oblique plane at the level where the femoral neck is narrowest. The angle is formed by two intersecting lines: the longitudinal axis of the femoral neck and from the isocentre of the femoral head to the point where sphericity of the femoral head is first lost [2] (Figure 21A). In clinical practice the routine measurement of the alpha angle is controversial as the cam morphology may occur in any part of the femoral head, and a normal measurement may be misleading. The femoral head-neck offset is also measured on the axial oblique plane at the level where the femoral neck is narrowest. Two parallel lines are drawn along the anterior aspect of the femoral head and neck. An offset of $<10 \mathrm{~mm}$ is consistent with cam-type morphology (Figure 21B). With cam FAI, during internal rotation and abduction of the hip, the aspherical (cam) portion of the femoral head enters into the joint, resulting in shearing forces and "outside-in" abrasion of the articular cartilage of the acetabulum. As a consequence, delamination of the articular cartilage may occur $[5,50]$. The acetabular cartilage lesions in cam FAI are usually more extensive than in pincer FAI due to the shearing forces inside the joint [51].

\section{Pincer morphology}

Pincer FAI refers to local or diffuse acetabular over-coverage contacting a normal femur, and typically affects middle-aged women. The centre-edge angle of Wiberg is determined on an anteroposterior (AP) radiograph of the hip and is formed by a line through the centre of the femoral head and the lateral end of the sclerotic weight-bearing area of the acetabulum (sourcil). A measurement of $<20^{\circ}$ indicates under-coverage (dysplastic hip), $20-25^{\circ}$ indicates borderline under-coverage, $25-39^{\circ}$ is normal coverage, and $\geq 40^{\circ}$ indicates over-coverage [52]. In the pincer type of impingent, there is linear impact between the excessive acetabular rim and femoral neck which compresses the labrum [51]. With pincer FAI, there is a deep acetabular socket (coxa profunda) and/or acetabular retroversion resulting in over-coverage of the femoral head. This results in mechanical conflict with linear impact between the acetabular rim and the femoral neck. In pincer FAI, the primary damage from the repetitive impact is upon the labrum followed by articular cartilage failure [4], unlike cam FAI. A small labrum is a common finding in pincer FAI. With chronicity, there is ossification of the acetabular rim, which further deepens the acetabular socket [53]. Pincertype impingement may be associated with labral ossifica- 

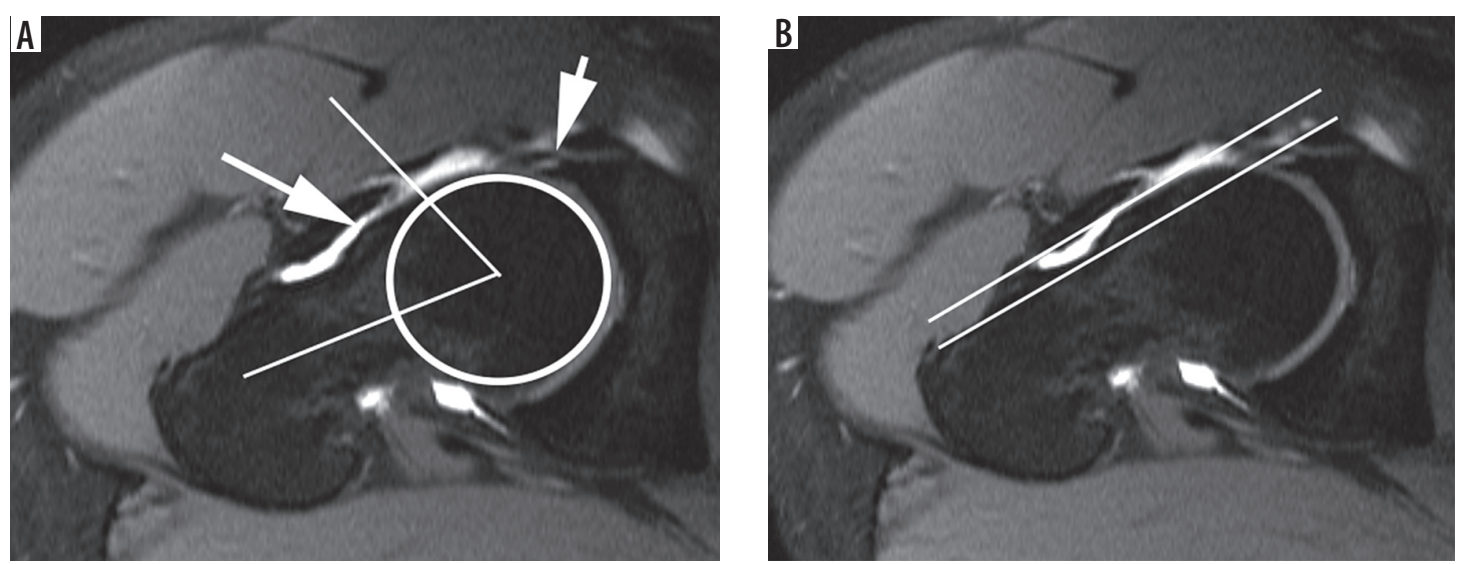

Figure 21. Cam morphology demonstrated on axial oblique T1W fat-saturated magnetic resonance arthrogram images of the hip. A) Axial oblique plane at the level where the femoral neck is narrowest. Note bone protuberance at the anterior aspect of the femoral head and neck junction consistent with cam deformity (long arrow). The alpha angle is formed by two intersecting lines: the longitudinal axis of the femoral neck and from the isocentre of the femoral head to the point where sphericity of the femoral head is first lost. An angle greater than 55 degrees is considered abnormal. There is an anterior acetabular labral tear (short arrow). B) Femoral head-neck offset is also measured on the axial oblique plane at the level where the femoral neck is narrowest. Two parallel lines are drawn along the anterior aspect of the femoral head and neck. An offset of $<10 \mathrm{~mm}$ is consistent with cam type morphology

tion, os acetabuli, or rim fractures, which may be due to abnormal development or stress fractures due to repetitive impingement [54]. Computed tomography may allow better delineation of the bony anatomy than MRI (Figure 22).

Compared to cam FAI, the articular cartilage lesions in pincer FAI are less pronounced and tend to be limited to the acetabular rim. Articular cartilage damage may also occur postero-inferiorly, which has been described as a contrecoup lesion. Specific examples of pincer morphology include congenital acetabular retroversion, os acetabuli, osteophytes, coxa profunda, and protrusio acetabuli, and these are usually well seen on axial or coronal plane MR images (Figure 23).

\section{Femoral torsion abnormalities}

Cam and pincer morphology have been considered the main predisposing factors to FAI, but in recent years there has been increasing recognition that femoral torsion abnor- malities may be an important factor [51,55,56]. Femoral torsion is the angle between the longitudinal axis of the femoral neck and the tangent at the condyles of the distal femur. The femoral neck usually points anteriorly in relation to the femoral condyles, hence the term femoral antetorsion. Normal femoral antetorsion is 13 degrees with a standard deviation of 10 degrees. Decreased femoral antetorsion reduces internal rotation and has been shown to be associated with camtype FAI. It has been suggested that all patients undergoing MRI assessment for FAI should have femoral antetorsion measurements performed, but this is not widely practiced. Recently, researchers have measured the supra- and infratrochanteric contribution to femoral torsion separately and showed that this is technically feasible [55].

\section{Subspine impingement}

Subspine or AIIS impingement results from abnormal contact between the AIIS and the proximal femur. It is an
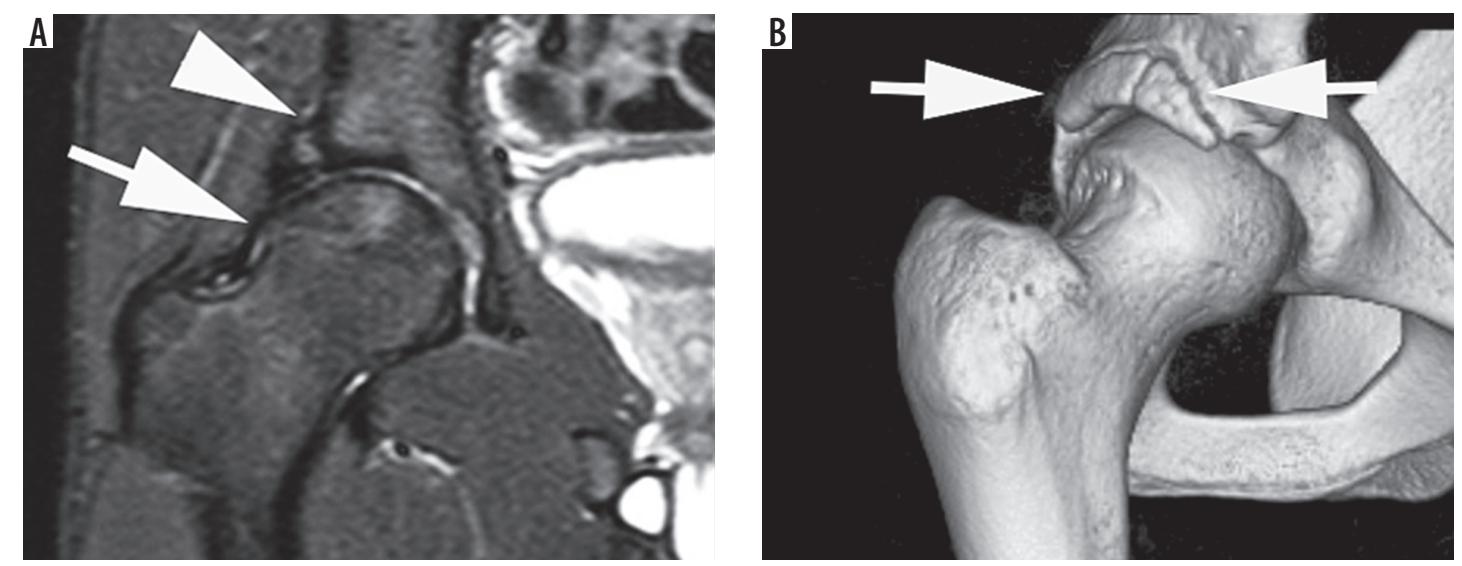

Figure 22. Mixed cam and pincer femoroacetabular impingement with acetabular rim fracture. A) Coronal proton density fat-saturated magnetic resonance image demonstrating a chronic acetabular rim fracture (short arrow) with over-coverage of the femoral head and cam morphology (long arrow). There is minor subarticular stress change with mild patchy bone marrow oedema at the periphery of the acetabulum and at the proximal aspect of the femoral head. B) 3D computed tomography reformat demonstrating the chronic acetabular rim fracture configuration (arrows) 


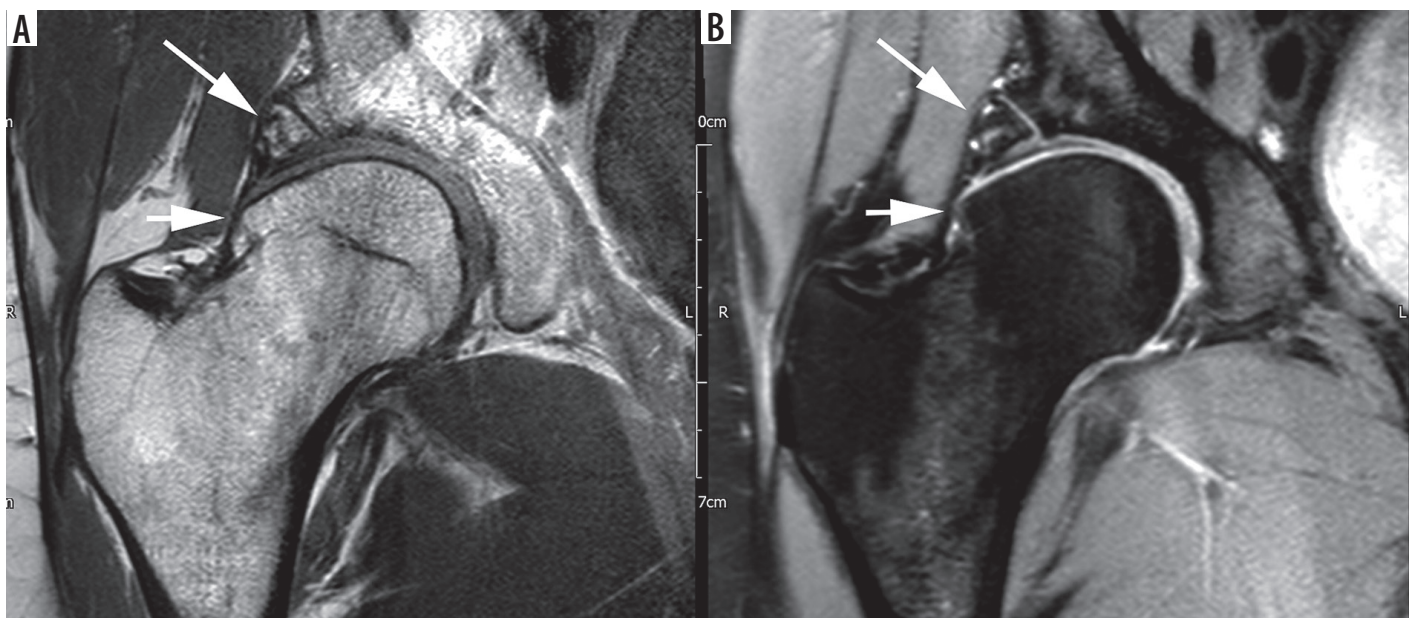

Figure 23. 0s acetabuli. A) Coronal T1W magnetic resonance (MR) and (B) proton density fat-saturated MR images demonstrating a large os acetabuli (long arrow) and cam morphology of the femoral head and neck junction (short arrow). The acetabular labrum is hypotrophic

under-recognised form of hip impingement, which can be a significant source of symptoms, and may be the cause of continuing pain after FAI surgery [57]. Three morphological AIIS variants have been described: Type I when there is a smooth ilium wall between the AIIS and the acetabular rim, Type II when the AIIS extends to the level of the rim, and Type III when the AIIS extends distally to the acetabular rim [58]. In patients with clinically and surgically diagnosed subspine impingement, as well as morphological features of the anterior inferior iliac spine, there may be cam deformity of the femoral head, signs of impingement in the proximal femur, and superior capsular oedema [59] (Figure 24).

\section{Hip dysplasia}

Hip dysplasia is defined as acetabular deficiency that causes instability and results in damage to intra-articular structures including the labrum, cartilage, and ligamentum teres.

Known complications of hip dysplasia include subchondral fractures because of weight-bearing forces being distributed over a smaller area and early OA due to labral and chondral injuries. MRI provides morphologic information about the acetabular deficiency and femoral neck-shaft angle, and it allows evaluation of associated intra-articular injuries and complications [60]. The acetabular deficiency may be quantified by measuring the centre-edge angle on anterior to middle images on coronal sequences, although it is technically a radiographic measurement, with the measurements on both modalities showing strong correlation [61]. Dysplastic hips have a centre-edge angle of Wiberg of less than 20 degrees. Hip dysplasia may be associated with coxa valga, which is a femoral neck and shaft angle of greater than 135 degrees [60]. Typical MRI findings are anterosuperior labral tears and hypertrophy with adjacent cartilage lesions, and ligamentum teres elongation, degeneration, and tearing (Figure 17).

\section{Osteoarthritis}

Osteoarthritis is the most common disease of the hip joint in adults. OA is a non-inflammatory degenerative disorder of the joint associated with cartilage degeneration. The articular cartilage thinning and defects typically affect the weightbearing areas. OA may be idiopathic or secondary to trauma, ON, inflammatory arthropathy, septic arthritis, ON, FAI,
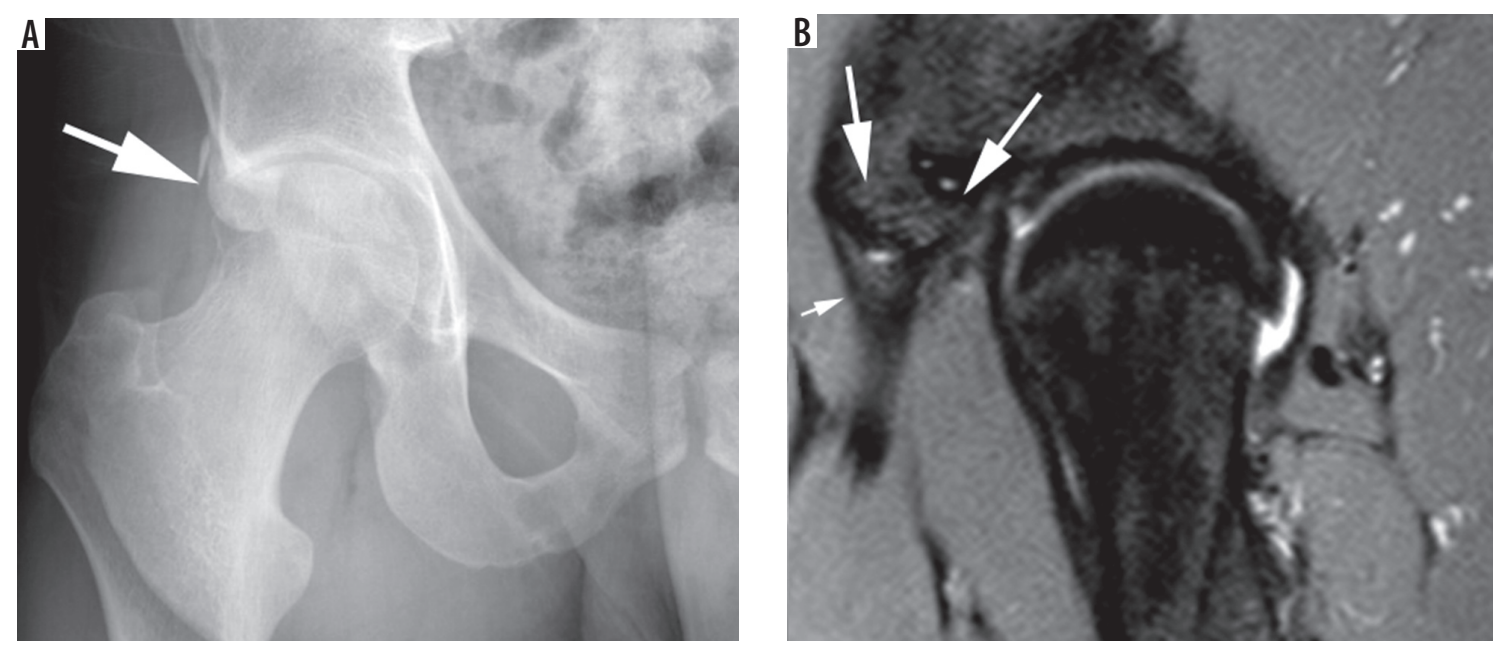

Figure 24. Subspine impingement. A) Anteroposterior radiograph of the hip demonstrating a type 3 anterior inferior iliac spine (AllS) configuration extending below the level of the acetabular rim following an old avulsion injury (arrow). B) Sagittal proton density fat-saturated magnetic resonance image demonstrating a malunited AllS (large arrows). The small arrow indicates the rectus femoris tendon 
hip dysplasia, Legg-Calve-Perthes disease, and SCFE. The classical radiographic findings of superior joint space loss, osteophyte formation, subchondral sclerosis, and cyst-like formation are well recognised. However, radiographs may appear relatively normal in early OA. Under these situations, MRI may be useful to determine if there is significant underlying hip pathology. MRI has the advantage of being able to directly visualise the articular cartilage. Other features of OA on MRI include detection of labral abnormalities, subchondral bone marrow oedema and cyst-like formation, and joint effusions. There may also be features of FAI, which is an established cause for premature OA (Figure 25).

Rapidly destructive OA of the hip is a less known entity characterised by rapid chondrolysis without evidence of other forms of destructive arthropathy. The destruction of the hip joint occurs within months of the onset of symptoms. The patients are predominantly women in their 60 s to 70 s with rapid clinical deterioration. The diagnosis is based on quick progression of hip pain and rapid chondrolysis on imaging. MRI features include extensive bone marrow oedema-like pattern in the femoral head and neck or acetabulum, subchondral cyst-like changes, insufficiency fracture with articular surface flattening or collapse, and non-specific joint effusion [62].

\section{Inflammatory arthropathy}

The hip joint is commonly involved in patients who have an inflammatory arthropathy, such as rheumatoid arthritis or ankylosing spondylitis. The hip joint may also be affected in psoriatic arthritis, juvenile idiopathic arthritis, polymyalgia rheumatica, and crystal deposition arthritis (Figure 26). MRI is the imaging modality of choice for the detection of early inflammation of the hip joints [27]. On MRI, the features of inflammatory arthropathy include joint effusion, synovial thickening, and bone marrow oedema [6]. Subchondral and periarticular bone marrow oedema or osteitis is a strong predictor for developing erosions [27]. Other features include bursitis, enthesitis, and cartilage damage. Cartilage thinning is diffuse in inflammatory arthritis, affecting the entire cartilage surface. Intravenous contrast may be useful to differentiate between joint effusions and synovitis. MRA is not recommended as joint effusion, and synovitis can be missed in the presence of intra-articular contrast [27]. A large hip effusion may decompress through the weak anterior capsule into the iliopsoas bursa. MRI also plays a crucial role in the follow-up of these conditions, to assess treatment response and identify complications [27].

\section{Septic arthritis}

Infection of the hip joint can occur as a result of haematogenous spread, direct inoculation or spread from the spine along the iliopsoas muscle. On MRI, septic arthritis may be indistinguishable from non-infective inflammatory arthropathy because of common features such

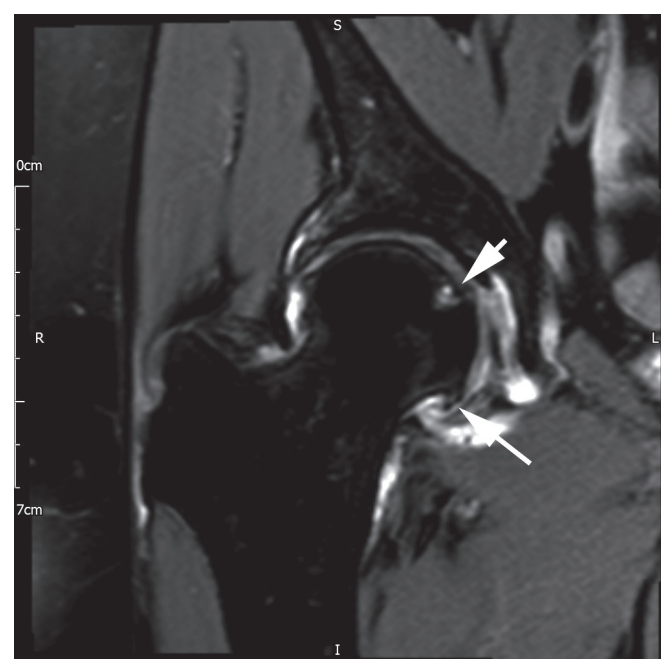

Figure 25. Hip osteoarthritis. Coronal proton density fat-saturated magnetic resonance image demonstrating substantial articular cartilage loss, a joint effusion, and prominent marginal osteophytes (long arrow) with early subchondral cyst-like formation in the femoral head (short arrow)

as synovial enhancement and joint effusion (Figure 27). However, joint infection should be suspected in the presence of adjacent soft tissue collections, sinus tracts, or osteomyelitis. Concomitant osteomyelitis of septic arthritis may be hard to differentiate from reactive bone marrow oedema, but the abnormal marrow signal of osteomyelitis is said to be more distinct as a low signal on T1-weighted images. The periarticular soft tissue oedema is usually more extensive in infection compared to non-infectious inflammation [27].

\section{Synovial chondromatosis}

Synovial chondromatosis can be divided into primary or secondary forms. Primary synovial chondromatosis is a benign process that is characterised by chondroid metaplasia in the sub-synovium forming multiple chondroid nodules that subsequently detach and result in intraarticular bodies within the joint, bursa, or tendon sheath. The chondral loose bodies are of fairly uniform size and shape, and eventually ossify. Synovial chondromatosis culminates in premature OA. The hip is the second most commonly affected joint after the knee [63]. Patients with primary synovial chondromatosis are typically men in the third to fifth decades of life [63] presenting with pain, swelling, and limitation of movement, which often progresses insidiously for years. On MRI, the signal intensity of the intra-articular bodies is variable, depending on the extent of mineralisation and ossification. Purely cartilaginous lesions are intermediate signal on T2-weighted sequences whereas calcified bodies appear as intermediate or low signal on all sequences. In late stage disease, fat marrow signal may be seen in the ossified loose bodies. Synovial hypertrophy returns high signal intensity on fluid-sensitive sequences and intermediate signal intensity on T1-weighted sequences. There may be extrinsic bone 


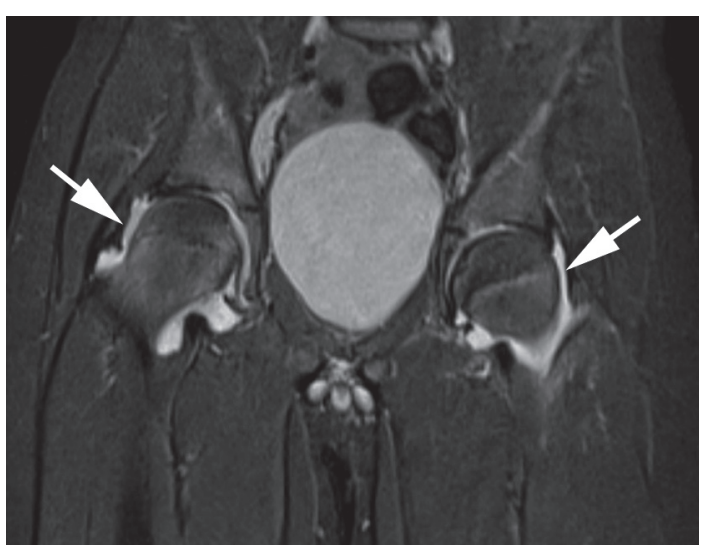

Figure 26. Bilateral hip synovitis in a teenager with juvenile idiopathic arthritis. Coronal short-tau inversion recovery magnetic resonance image demonstrating hyperintense synovial thickening (arrows) of both hips

erosion in 20 to $50 \%$ of cases, resulting in an "apple core" deformity of the femoral neck [63] (Figure 28). Secondary synovial chondromatosis may arise as a result of joint abnormalities, most commonly OA. The intra-articular bodies associated with secondary synovial chondromatosis tend to be fewer with less uniform size and shape when compared to those occurring with primary synovial chondromatosis.

\section{Tenosynovial giant cell tumour}

Tenosynovial giant cell tumour, commonly referred to as pigmented villonodular synovitis (PVNS), may be localised or diffuse. When the hip is involved it is most commonly affected by the diffuse form [64]. Translocations of the colony-stimulating factor 1 (CSF-1) gene are present in PVNS, resulting in over-expression of CSF and intra-articular haemorrhage with hemosiderin deposition. Imaging is important for the diagnosis, surgical planning, and follow-up. Radiographs are often normal in the early stages of disease. MRI is the examination of choice because it accurately depicts the extent and location of disease, and is also very useful for detecting disease recurrence after synovectomy [65]. On MRI there is typically a joint effusion with low T1 and T2 signal synovial thickening indicating hemosiderin deposition (Figure 29). On gradient echo sequences there may be associated blooming artefact due to the hemosiderin. As the disease progresses bone erosions may occur, resulting in erosion of the femoral neck, and subchondral cyst-like changes.

\section{Extra-articular pathology}

\section{Ischiofemoral impingement}

Ischiofemoral impingement is an extra-articular soft tissue impingement between the lesser trochanter of the proximal femur and the ischium, and it is an uncommon cause of chronic hip and buttock pain. It is characterised by narrowing of the ischiofemoral and quadratus femoris spaces with resulting damage to the quadratus femoris muscle. On MRI, there is signal abnormality in the quadratus femoris muscle seen as oedema in the acute phase and fatty infiltration with wasting if the compression persists for an extended period of time [66] (Figure 30). The ischiofemoral and quadratus femoris spaces are measured on an axial image, defined as the smallest distance between the lateral cortex of the ischial tuberosity and medial cortex of the lesser trochanter and the smallest space for the passage of the quadratus femoris muscle delimited by the superolateral surface of the hamstring tendons and the posteromedial surface of the iliopsoas tendon or lesser trochanter, respectively [67]. Several studies have found that the measured ischiofemoral and quadratus femoris spaces were significantly different between symptomatic and control groups. The mean ischiofemoral and quadratus femoris distances in symptomatic groups ranged from 13 to $17 \mathrm{~mm}$ and
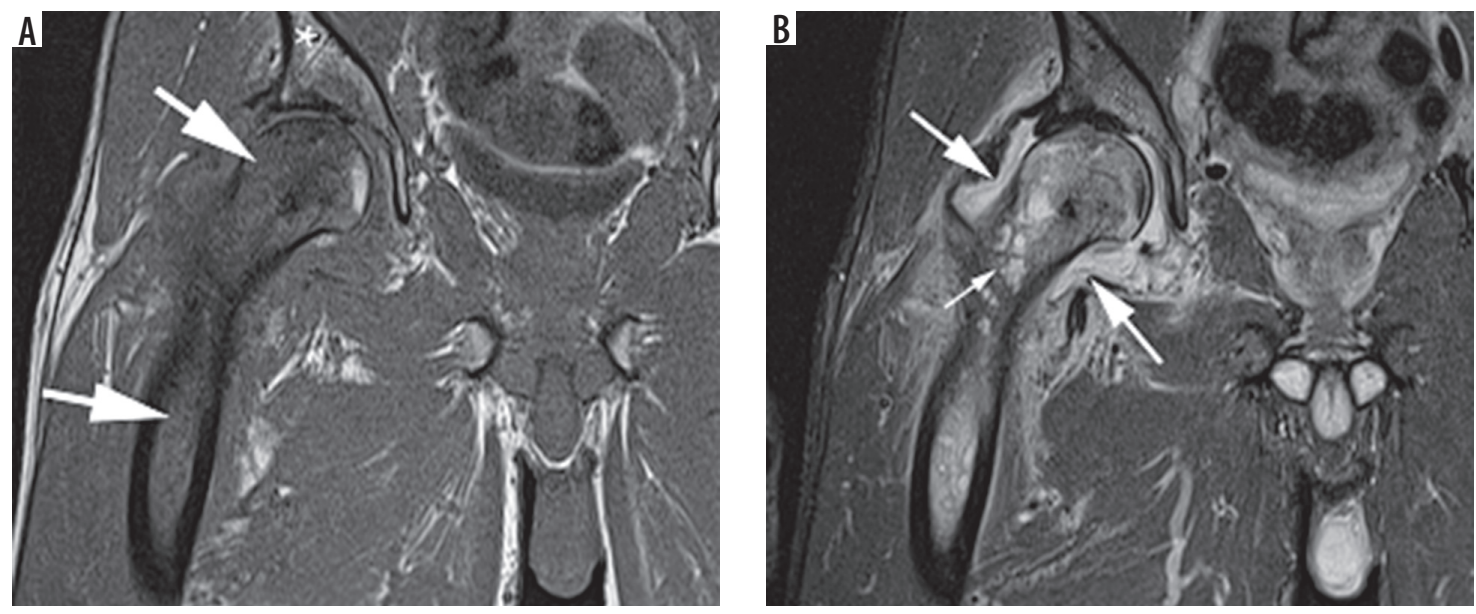

Figure 27. Septic arthritis and osteomyelitis involving the right hip in an intravenous drug user. A) Coronal T1W magnetic resonance (MR) image demonstrating extensive low T1 bone marrow signal in the proximal femur indicating osteomyelitis (arrows). Note normal bone marrow signal in the acetabulum (asterisk). B) Coronal short-tau inversion recovery MR demonstrating a large hip joint effusion with marked synovitis (large arrows) and extensive heterogeneous abnormal signal in the proximal right femur (small arrow) indicating osteomyelitis 


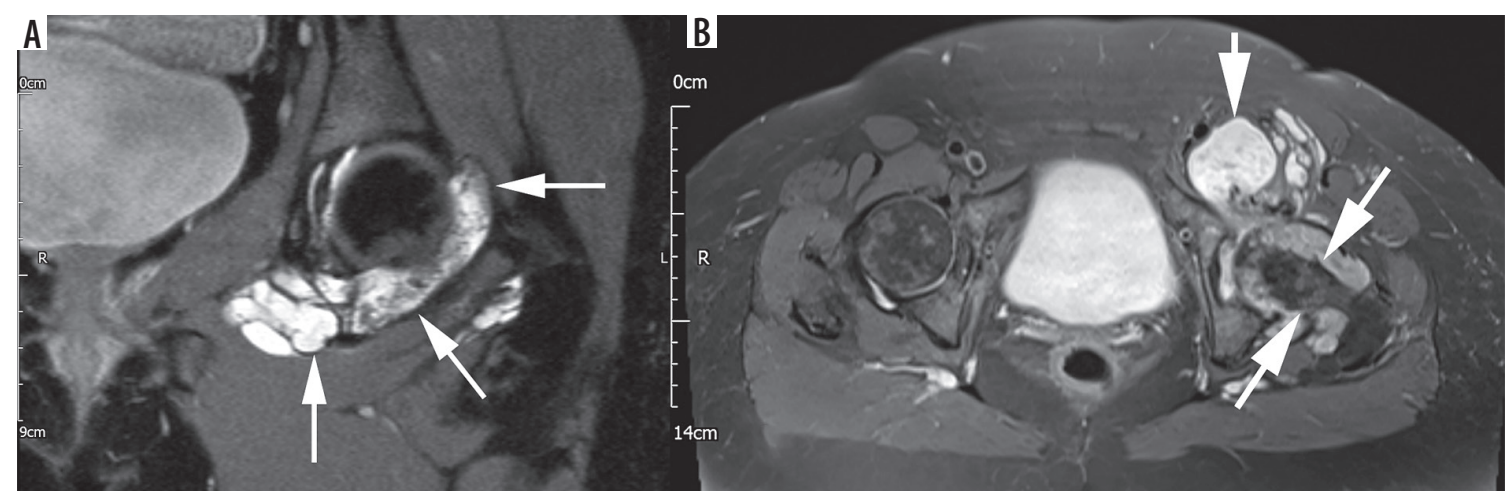

Figure 28. Synovial chondromatosis. A) Coronal proton density (PD) fat-saturated (FS) magnetic resonance (MR) image demonstrating synovitis (arrows) with multiple low signal foci in keeping with mineralised joint bodies. B) Axial PD FS MR image demonstrating florid synovial hypertrophy with distension of the iliopsoas bursa (short arrow). Note heterogeneous signal within the synovium suggesting the diagnosis of synovial chondromatosis. There is severe erosion of the femoral neck resulting in a classical "apple core" deformity (long arrows)

from 7 to $12 \mathrm{~mm}$, respectively [67,68]. The narrowed ischiofemoral and quadratus femoris spaces may be congenital with a female preponderance, or acquired from intertrochanteric fractures, hip surgery including arthroplasty or osteotomy, hip joint OA with superior and medial migration of the femur, hamstring enthesopathy, and osteochondroma $[67,69]$. The condition may occur secondarily to injury in other muscles controlling movement of the hip, such as the hamstrings and gluteus medius [66]. There may be an adjacent bursa-like formation that reflects chronic impingement.

\section{Snapping hip}

The snapping hip is a symptom complex characterised by an audible or palpable snapping of the hip during motion, which is accompanied by discomfort. This condition is common among athletes and dancers [6]. Snapping hip syndrome may be a result of external, internal, or intra-articular causes. The external snapping hip is the most common and is usually caused by restrain of the iliotibial band or the anterior edge of the gluteus maximus muscle over the greater trochanter during flexion and extension of the hip until it suddenly releases and moves anteriorly over the lateral facet causing the snap. Internal snapping of the hip is caused by abnormal iliopsoas tendon movement on the iliopectineal eminence or the lesser trochanter [2]. Iliopsoas impingement may also occur at the anterior acetabular rim and can be associated with an acetabular labral tear at the three oclock position (Figure 31). Dynamic ultrasound is considered the best modality for evaluating external and internal snapping hip because it allows evaluation of the muscles and tendon during range of motion. However, MRI may demonstrate thickening of the tendon, surrounding soft tissue oedema, and bursal fluid on fluid-sensitive images. Intra-articular snapping may be caused by labral tear or intra-articular loose bodies including synovial chondromatosis. MRI and MRA are the investigations of choice for intra-articular snapping hip.

\section{Greater trochanter pain syndrome}

Greater trochanteric pain syndrome (GTPS) is common and is characterised by lateral hip pain and tenderness that encompasses pathologies of the gluteal and short external rotator tendons, iliotibial band, and trochanteric bursa [21]. The most common underlying cause for GTPS is gluteus minimus and medius insertional tendinosis with or without tear from chronic repetitive microtrauma [2], and this may or may not be associated with trochanteric bursitis (Figure 32). The patient is classically a middle-aged female although both genders can be affected at any age [21]. While ultrasound can be used to evaluate the soft tissue structures about the greater trochanter, it is operator
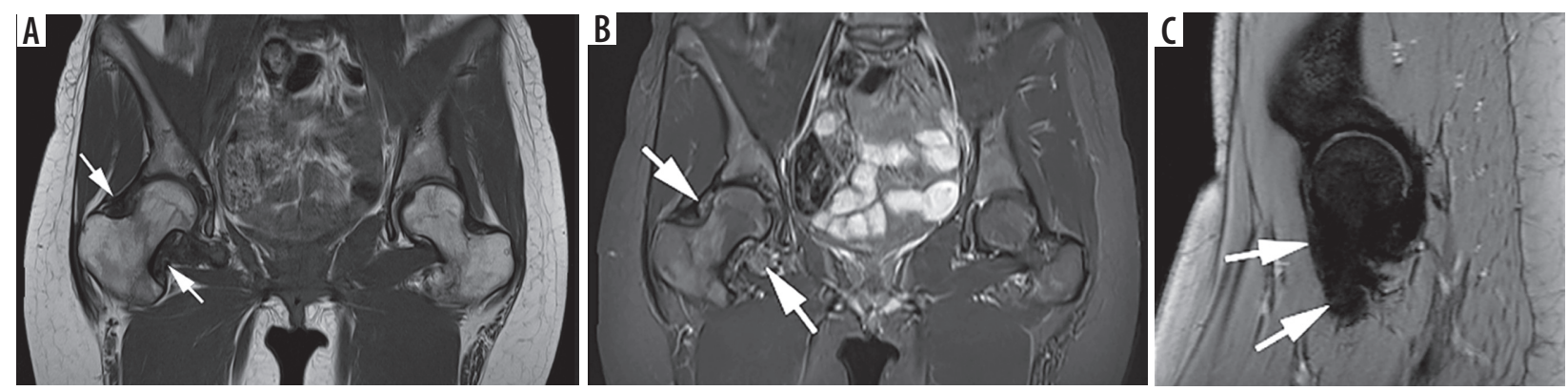

Figure 29. Tenosynovial giant cell tumour of the right hip. Coronal (A) T1W and (B) short-tau inversion recovery magnetic resonance (MR) images demonstrating low signal synovial hypertrophy at the right hip (arrows). C) Sagittal gradient echo MR image demonstrating "blooming artefact" due to hemosiderin deposition (arrows) 


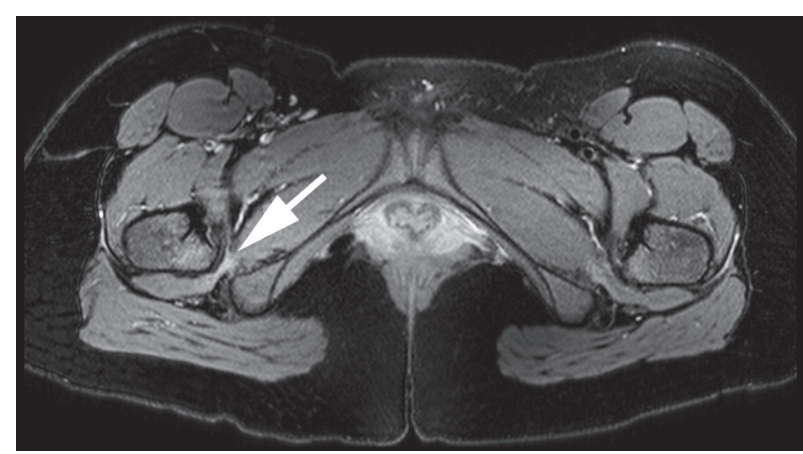

Figure 30. Ischiofemoral impingement. Axial short-tau inversion recovery magnetic resonance image demonstrating narrowing of the right ischiofemoral and quadratus femoris spaces with oedema in the quadratus femoris muscle (arrow). There is also minor narrowing of the left ischiofemoral space

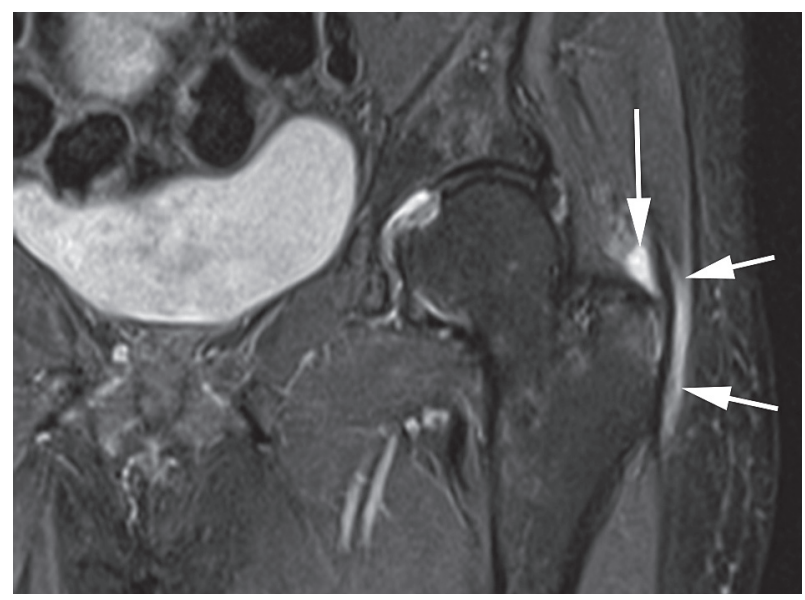

Figure 32. Trochanteric bursitis. Coronal short-tau inversion recovery magnetic resonance image demonstrating distension of the greater trochanteric (short arrows) and subgluteus medius (long arrow) bursae consistent with bursitis

dependent, and MRI shows higher correlation with clinical and intraoperative findings [21]. On MRI, tendinosis is characterised by thickening and signal alteration of the tendon. Eventually, partial or complete tears may occur (Figure 33). Chronic tendinosis is associated with bony changes at the greater trochanter where the tendon inserts, such as cortical irregularity, enthesophytes, sclerosis, and small cysts. Calcific deposits within the tendon may be easily overlooked on MRI. Peri-trochanteric fluid signal is evident in GTPS but is also frequently encountered in the asymptomatic population, so this finding should be interpreted in the clinical context. Ultrasound-guided injection of corticosteroid and local anaesthetic into the trochanteric bursa provides pain relief and helps facilitate physiotherapy and rehabilitation [2].

GTPS may develop as a complication following hip arthroplasty, and this is thought to be dependent on the surgical approach and the associated soft tissue damage. The lateral approach is associated with damage to the hip abductor muscles and tendons, while the posterior approach is associated with damage to the short external rotator muscles and tendons $[21,70]$. The anterior and anterolateral approaches are intermuscular approaches thus cause less soft tissue damage. Identification of the surgical approach after hip arthroplasty

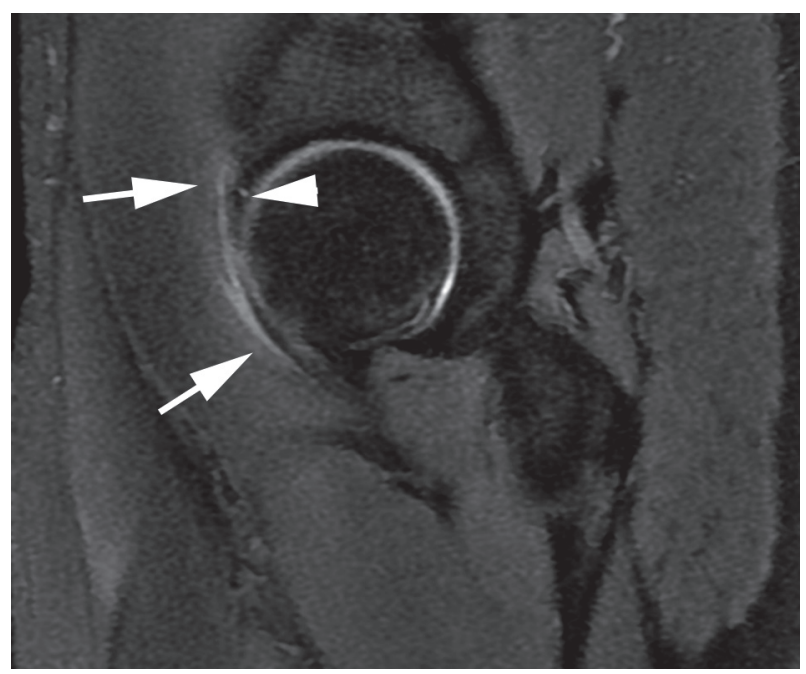

Figure 31. Iliopsoas impingement with anterior labral tear in a 17-yearold female with atraumatic anterior right hip pain. Sagittal proton density fat-saturated magnetic resonance image demonstrating oedema around the iliopsoas tendon (arrows) with a small fluid cleft indicating a tear in the anterior acetabular labrum at 3:00 position (arrowhead)

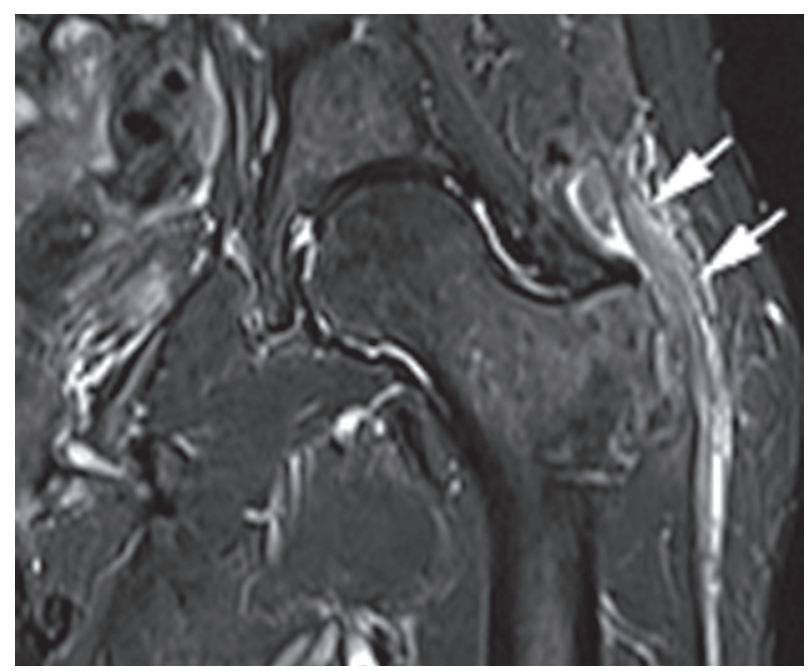

Figure 33. Severe gluteus medius tendinosis. Coronal short-tau inversion recovery magnetic resonance image demonstrating thickening and altered increased signal of the left gluteus medius tendon in keeping with severe tendinosis with interstitial partial-thickness tearing. Note surrounding soft tissue oedema

is possible with MRI [70], and awareness of the types of surgical access is useful for the radiologist when evaluating the structures at the greater trochanter.

\section{Conclusions}

The radiologist should be aware of commonly encountered painful conditions of the hip and be familiar with the characteristic findings on MRI. Detailed knowledge of the anatomy of the hip joint and its surrounding structures is essential in understanding hip disorders.

\section{Conflict of interest}

The authors report no conflict of interest. 


\section{References}

1. Omoumi P, Vande Berg B. Hip imaging: normal variants and asymptomatic findings. Semin Musculoskelet Radiol 2017; 21: 507-517.

2. Hegazi TM, Belair JA, McCarthy EJ, et al. Sports injuries about the hip: what the radiologist should know. Radiographics 2016; 36: 1717-1745.

3. Chang CY, Huang AJ. MR imaging of normal hip anatomy. Magn Reson Imaging Clin N Am 2013; 21: 1-19.

4. Ladd LM, Blankenbaker DG, Davis KW, et al. MRI of the hip: important injuries of the adult athlete. Curr Radiol Rep 2014; 2: 51123.

5. Blankenbaker DG, Tuite MJ. The painful hip: new concepts. Skeletal Radiol 2006; 35: 352-370.

6. Teh J. Imaging the hip. Imaging 2007; 19: 234-248.

7. Schmaranzer F, Todorski IAS, Lerch TD, et al. Intra-articular lesions: imaging and surgical correlation. Semin Musculoskelet Radiol 2017; 21: 487-506.

8. Agten CA, Sutter R, Buck FM, et al. Hip imaging in athletes: sports imaging series. Radiology 2016; 280: 351-369.

9. Ilizaliturri VM, Byrd JWT, Sampson TG, et al. A geographic zone method to describe intra-articular pathology in hip arthroscopy: cadaveric study and preliminary report. Arthroscopy 2008; 24: 534-539.

10. Naraghi A, White LM. MRI of labral and chondral lesions of the hip. Am J Roentgenol 2015; 205: 479-490.

11. Sutter R, Zubler V, Hoffmann A, et al. Hip MRI: how useful is intraarticular contrast material for evaluating surgically proven lesions of the labrum and articular cartilage? Am J Roentgenol 2014; 202: 160-169.

12. Magee T. Comparison of 3.0-T MR vs. 3.0-T MR arthrography of the hip for detection of acetabular labral tears and chondral defects in the same patient population. Br J Radiol 2015; 88: 1-7.

13. Llopis E, Cerezal L, Kassarjian A, et al. Direct MR arthrography of the hip with leg traction: feasibility for assessing articular cartilage. Am J Roentgenol 2008; 190: 1124-1128.

14. Schmaranzer F, Klauser A, Kogler M, et al. Diagnostic performance of direct traction MR arthrography of the hip: detection of chondral and labral lesions with arthroscopic comparison. Eur Radiol 2015; 25: 1721-1730.

15. Vilanova JC, Ribes R. Learning diagnostic imaging. In: Musculoskeletal imaging. Berlin, Heildelberg: Springer; 2008; 127-151.

16. Cerezal L, Kassarjian A, Canga A, et al. Anatomy, biomechanics, imaging, and management of ligamentum teres injuries. Radiographics 2010; 30: 1637-1651

17. Blankenbaker DG, de Smet AA, Keene JS, et al. Imaging appearance of the normal and partially torn ligamentum teres on hip MR arthrography. Am J Roentgenol 2012; 199: 1093-1098.

18. Wagner F V, Negrão JR, Campos J, et al. Capsular ligaments of the hip: anatomic, histologic, and positional study in cadaveric specimens with MR arthrography. Radiology 2012; 263: 189-198.

19. Robinson P, White LM, Agur A, et al. Obturator externus bursa: anatomic origin and MR imaging features of pathologic involvement Radiology 2003; 228: 230-234.

20. Pfirrmann CWA, Chung CB, Theumann NH, et al. Greater trochanter of the hip: Attachment of the abductor mechanism and a complex of three bursae - MR imaging and MR bursography in cadavers and MR imaging in asymptomatic volunteers. Radiology 2001; 221: 469-477.
21. Hirschmann A, Falkowski AL, Kovacs B. Greater trochanteric pain syndrome: abductors, external rotators. Semin Musculoskelet Radiol 2017; 21: 539-546.

22. Kassarjian A, Fritz B, Afonso PD, et al. Guidelines for MR. Imaging of the hip region. 2016. Available at: https:/essr.org/content-essr/uploads/2016/10/ESSR_Sports_guidelines.pdf.

23. Sheehan SE, Shyu JY, Weaver MJ, et al. Proximal femoral fractures: what the orthopedic surgeon wants to know. Radiographics 2015; 35: 1563-1584.

24. Long MM, Stetts DM. Stress fractures of the femoral neck. Orthop Nurs 1985; 4: 70-72.

25. Kim SJ, Ahn J, Kim HK, et al. Is magnetic resonance imaging necessary in isolated greater trochanter fracture? A systemic review and pooled analysis. BMC Musculoskelet Disord 2015; 16: 395.

26. Ikemura S, Yamamoto T, Motomura G, et al. MRI evaluation of collapsed femoral heads in patients 60 years old or older: Differentiation of subchondral insufficiency fracture from osteonecrosis of the femoral head. Am J Roentgenol 2010; 195: W63-68.

27. Schueller-Weidekamm C, Teh J. Inflammatory conditions of the hip. Semin Musculoskelet Radiol 2017; 21: 589-603.

28. Murphey MD, Foreman KL, Klassen-Fischer MK, et al. From the radiologic pathology archives: imaging of osteonecrosis: radiologic-pathologic correlation. Radiographics 2014; 34: 1003-1028.

29. Mitchell DG, Steinberg ME, Dalinka MK, et al. Magnetic resonance imaging of the ischemic hip. Alterations within the osteonecrotic, viable, and reactive zones. Clin Orthop Relat Res 1989; 244: 60-77.

30. Jawad MU, Haleem AA, Scully SP. In brief: Ficat classification: avascular necrosis of the femoral head. Clin Orthop Relat Res 2012; 470: 2636-2639.

31. Lee GC, Khoury V, Steinberg D, et al. How do radiologists evaluate osteonecrosis? Skeletal Radiol 2014; 43: 607-614.

32. Ficat RP. Idiopathic bone necrosis of the femoral head. Early diagnosis and treatment. J Bone Joint Surg Br 1985; 67: 3-9.

33. Lamer S, Dorgeret S, Khairouni A, et al. Femoral head vascularisation in Legg-Calvé-Perthes disease: Comparison of dynamic gadolinium-enhanced subtraction MRI with bone scintigraphy. Pediatr Radiol 2002; 32: 580-585.

34. Ducou le Pointe H, Haddad S, Silberman B, et al. Legg-Perthes-Calvé disease: staging by MRI using gadolinium. Pediatr Radiol 1994; 24: 88-91.

35. Merlini L, Combescure C, de Rosa V, et al. Diffusion-weighted imaging findings in Perthes disease with dynamic gadolinium-enhanced subtracted (DGS) MR correlation: a preliminary study. Pediatr Radiol 2010; 40: 318-325.

36. De Rosa V, Laurent M, Canavese F, et al. A simple, precocious, and reliable way to assess future clinical outcome in children with Perthes disease and mild femoral head involvement: correlation between MRI with diffusion-weighted and dynamic gadolinium-enhanced subtraction and Catterall and Herring classifications. Eur J Orthop Surg Traumatol 2018; 28: 1283-1290.

37. Szwedowski D, Nitek Z, Walecki J. Evaluation of transient osteoporosis of the hip in magnetic resonance imaging. Polish J Radiol 2014; 79: 36-38 
38. Balakrishnan A, Schemitsch EH, Pearce D, et al. Distinguishing transient osteoporosis of the hip from avascular necrosis. Can J Surg 2003; 46: 187-192.

39. Gray AJR, Villar RN. The ligamentum teres of the hip: an arthroscopic classification of its pathology. Arthroscopy 1997; 13: 575-578.

40. McCarthy JC, Noble PC, Schuck MR, et al. The role of labral lesions to development of early degenerative hip disease. Clin Orthop Relat Res 2001; 393: 25-37.

41. Schmaranzer F, Todorski IAS, Lerch TD, et al. Intra-articular lesions: imaging and surgical correlation. Semin Musculoskelet Radiol 2017; 21: 487-506.

42. Blankenbaker DG, de Smet AA, Keene JS, et al. Classification and localization of acetabular labral tears. Skeletal Radiol 2007; 36: 391-397.

43. Freedman BA, Potter BK, Dinauer PA, et al. Prognostic value of magnetic resonance arthrography for czerny stage II and III acetabular labral tears. Arthroscopy 2006; 22: 742-747.

44. Czerny C, Hofmann S, Urban M, et al. MR arthrography of the adult acetabular capsular-labral complex: Correlation with surgery and anatomy. Am J Roentgenol 1999; 173: 345-349.

45. Neumann G, Mendicuti AD, Zou KH, et al. Prevalence of labral tears and cartilage loss in patients with mechanical symptoms of the hip: evaluation using MR arthrography. Osteoarthr Cartil 2007; 15: 909-917.

46. Albers CE, Wambeek N, Hanke MS, et al. Imaging of femoroacetabular impingement-current concepts. J Hip Preserv Surg 2016; 3: 245-261.

47. Leunig M, Beck M, Kalhor M, et al. Fibrocystic changes at anterosuperior femoral neck: prevalence in hips with femoroacetabular impingement. Radiology 2005; 236: 237-246.

48. Nepple JJ, Vigdorchik JM, Clohisy JC. What is the association between sports participation and the development of proximal femoral cam deformity? Am J Sports Med 2015; 43: 2833-2840.

49. Nötzli HP, Wyss TF, Stoecklin CH, et al. The contour of the femoral head-neck junction as a predictor for the risk of anterior impingement. J Bone Joint Surg Br 2002; 84: 556-560.

50. Pfirrmann CWA, Duc SR, Zanetti M, et al. MR arthrography of acetabular cartilage delamination in femoroacetabular cam impingement. Radiology 2008; 249: 236-241.

51. Sutter R, Pfirrmann CWA. Update on femoroacetabular impingement: what is new, and how should we assess it? Semin Musculoskelet Radiol 2017; 21: 518-528.

52. Tannast M, Hanke MS, Zheng G, et al. What are the radiographic reference values for acetabular under- and overcoverage? Clin Orthop Relat Res 2015; 473: 1234-1246.

53. Corten K, Ganz R, Chosa E, et al. Bone apposition of the acetabular rim in deep hips: a distinct finding of global pincer impingement. J Bone Joint Surg Br 2011; 93: 10-16.

54. Larson CM, Stone RM. The rarely encountered rim fracture that contributes to both femoroacetabular impingement and hip stability: a report of 2 cases of arthroscopic partial excision and internal fixation. Arthroscopy 2011; 27: 1018-1022.

55. Fritz B, Bensler S, Leunig M, et al. MRI assessment of supra- and infratrochanteric femoral torsion: association with femoroacetabular impingement and hip dysplasia. Am J Roentgenol 2018; 211: 155-161.

56. Sutter R, Dietrich TJ, Zingg PO, et al. Femoral antetorsion: comparing asymptomatic volunteers and patients with femoroacetabular impingement. Radiology 2012; 263: 475-483.

57. Carton P, Filan D. Anterior inferior iliac Spine (AIIS) and subspine hip impingement. Muscles Ligaments Tendons J 2016; 6: 324-336.
58. Hetsroni I, Poultsides L, Bedi A, et al. Anterior inferior iliac spine morphology correlates with hip range of motion: A classification system and dynamic model hip. Clin Orthop Relat Res 2013; 471: 2497-2503.

59. Samim M, Walter W, Gyftopoulos S, et al. MRI assessment of subspine impingement: Features beyond the anterior inferior iliac spine morphology. Radiology 2019; 293: 412-421.

60. Beltran LS, Rosenberg ZS, Mayo JD, et al. Imaging evaluation of developmental hip dysplasia in the young adult. Am J Roentgenol 2013; 200: 1077-1088.

61. Chen L, Boonthathip M, Cardoso F, et al. Acetabulum protrusio and center edge angle: New MR-imaging measurement criteria a correlative study with measurement derived from conventional radiography. Skeletal Radiol 2009; 38: 123-129.

62. Boutry N, Paul C, Leroy X, et al. Original report of the hip: MR imaging findings. AJR Am J Roentgenol 2002; 179: 657-663.

63. Murphey MD, Vidal JA, Fanburg-Smith JC, et al. From the archives of the AFIP: imaging of synovial chondromatosis with radiologic-pathologic correlation. Radiographics 2007; 27: 1465-1488.

64. Steinmetz S, Rougemont AL, Peter R. Pigmented villonodular synovitis of the hip. EFORT Open Rev 2016; 1: 260-266.

65. Murphey MD, Rhee JH, Lewis RB, et al. From the archives of the AFIP pigmented villonodular synovitis: radiologic-pathologic correlation. Radiographics 2008; 28: 1493-1518.

66. Ali AM, Teh J, Whitwell D, et al. Ischiofemoral impingement: a retrospective analysis of cases in a specialist orthopaedic centre over a four year period. Hip Int 2013; 23: 263-268.

67. Torriani M, Souto SCL, Thomas BJ, et al. Ischiofemoral impingement syndrome: An entity with hip pain and abnormalities of the quadratus femoris muscle. Am J Roentgenol 2009; 193: 186-190.

68. Akça A, Safak KY, Ilis ED, et al. Ischiofemoral impingement: assessment of MRI findings and their reliability. Acta Ortop Bras 2016; 24: 318-321.

69. Yoong P, Mansour R, Teh JL. Multiple hereditary exostoses and ischiofemoral impingement: a case-control study. Skeletal Radiol 2014; 43: 1225-1230.

70. Agten CA, Sutter R, Dora C, et al. MR imaging of soft tissue alterations after total hip arthroplasty: comparison of classic surgical approaches. Eur Radiol 2017; 27: 1312-1321. 